\title{
LPS-induced lung platelet recruitment occurs independently from neutrophils, PSGL-1, and P-selectin.
}

Simon J. Cleary, ${ }^{1}$ Carl Hobbs, ${ }^{2}$ Richard T. Amison, ${ }^{1}$ Stephanie Arnold, ${ }^{1}$ Blaze G. O'Shaughnessy, ${ }^{1}$ Emma Lefrançais, ${ }^{3}$ Beñat Mallavia, ${ }^{3}$ Mark R. Looney, ${ }^{3}$ Clive P. Page, ${ }^{1}$ Simon C. Pitchford.$^{1 *}$

${ }^{1}$ Sackler Institute of Pulmonary Pharmacology, Institute of Pharmaceutical Science, King's College

London, London, UK.

${ }^{2}$ The Wolfson Centre for Age Related Diseases, King's College London, London, UK.

${ }^{3}$ University of California San Francisco (UCSF), Department of Medicine, San Francisco, USA

*Author for correspondence and reprint requests:

Dr Simon Pitchford

Sackler Institute of Pulmonary Pharmacology

Institute of Pharmaceutical Science

5.43 Franklin Wilkins Building

150 Stamford Street

Waterloo Campus

King's College London

London UK

SE1 9NH

Phone: +44 2078484266

Fax: +44 2078484788

Simon.pitchford@kcl.ac.uk

Running header: Neutrophil-independent lung platelet recruitment.

Authorship: S.J.C. designed and performed the research, analysed the data, and wrote the paper. C.H. contributed to assay development. R.T.A., S.A., B.G.O'S performed research. E.L., and B.M., performed research and contributed to assay development. M.R.L. designed research and contributed vital assay. C.P.P. designed research and helped write paper. S.C.P. proposed the project, designed research and wrote paper.

Funding Acknowledgement: This research was funded by a Medical Research Council Doctoral Training Grant (MRC-DTP), and associated in vivo strategic skills funds awarded to Dr Simon Pitchford to fund Simon Cleary; Sackler Foundation awards to Professor Clive Page, and travelling fellowships from the British Pharmacological Society and King's College London Physiology Department awarded to Simon Cleary. Work conducted in the laboratory of Professor Mark Looney was supported by NIH grant: NIH R01HL107386.

Conflict of interest. No author has a conflict of interest to disclose.

Keywords: Platelets, neutrophils, recruitment, lungs, LPS

Text word count: 5000, Abstract word count: 234, Number of figures: 5, Number of references: 53

Parts of this work have been presented in abstract form at the Winter Meeting of the British Pharmacological Society, London, UK, December 14, 2016, and the European Respiratory Congress, Milan, Italy, September 10, 2017. 


\section{Abstract}

Platelets are recruited to inflammatory foci and contribute to host defence and inflammatory responses. Compared to platelet recruitment in haemostasis and thrombosis, the mechanisms of platelet recruitment in inflammation and host defence are poorly understood. Neutrophil recruitment to lung airspaces following inhalation of bacterial LPS requires platelets and PSGL-1 in mice. Given this association between platelets and neutrophils, we investigated whether recruitment of platelets to lungs of mice following LPS inhalation was dependent on PSGL-1, P-selectin, or interaction with neutrophils.

BALB/c mice were administered intranasal LPS $(055: B 5,5 \mathrm{mg} / \mathrm{kg})$ and 48 hours later lungs were collected, and platelets and neutrophils were quantified in tissue sections by immunohistochemistry. The effects of functional blocking antibody treatments targeting the platelet-neutrophil adhesion molecules P-selectin, or PSGL-1, or treatment with a neutrophil depleting antibody targeting Ly6G, were tested on the extent of LPS-induced lung platelet recruitment. Separately in Pf4-CrexmTmG mice, two-photon intravital microscopy was used to image platelet adhesion in live lungs.

Inhalation of LPS caused both platelet and neutrophil recruitment to the lung vasculature. However, decreasing lung neutrophil recruitment by blocking PSGL-1, P-selectin, or depleting blood neutrophils had no effect on lung platelet recruitment. Lung intravital imaging revealed increased adhesion of platelets in the lung microvasculature which was not associated with thrombus formation.

In conclusion, platelet recruitment to lungs in response to LPS occurs through mechanisms distinct from those mediating neutrophil recruitment, or the occurrence of pulmonary emboli.

Keywords: Platelets, neutrophils, recruitment, lungs, LPS 


\section{Introduction}

Platelet and neutrophil recruitment to lungs are critical to host defence, but when excessive or inappropriate can also contribute to tissue damage in a range of inflammatory diseases including: acute respiratory distress syndrome (ARDS), chronic obstructive pulmonary disease (COPD), and respiratory infections. ${ }^{1,2}$ Research suggests that platelets can exert a controlling influence on neutrophil recruitment, particularly in acute inflammation in the lung, where responses are dependent upon the presence of platelets displaying adhesion molecules that interact with counterligands expressed on neutrophils, ${ }^{3-5}$ signalling in platelets through the purinergic receptors $P 2 Y_{1}$ and $\mathrm{P}_{2} \mathrm{Y}_{14},{ }^{6,7} \mathrm{P}-\mathrm{Rex} 1$ and Vav guanine nucleotide exchange factors, ${ }^{8}$ and the release of CCL5, CXCL4 and CXCL7 chemokines, ${ }^{9}$ and serotonin, ${ }^{10}$ from platelets. These data provide evidence of plateletdependent signalling mechanisms by which platelets influence neutrophil recruitment. Associations between platelets and neutrophils are further observed in acute lung inflammation by the presence of heightened circulating blood platelet-neutrophil complexes, ${ }^{11}$ and platelet dependence of both neutrophil rolling, intravascular crawling and early diapedesis. ${ }^{12}$ Furthermore, platelets can sometimes be found associated with extravascular neutrophils in inflamed lungs. ${ }^{11,13-15}$

How leukocytes are recruited from blood to tissues to act as major effectors of host defence and inflammation has become increasingly well characterised. ${ }^{2,16}$ The involvement of platelets in leukocyte recruitment in inflammation therefore adds a further point of control for coordinating inflammatory responses. ${ }^{1,17}$

Blood platelet recruitment to lesions in blood vessel walls is also becoming increasingly understood at a molecular level in the context of haemostasis and thrombosis. Similarly to leukocytes, platelets can independently respond to pathogen-associated molecular patterns, and importantly can be recruited from blood to accumulate in tissues following infection or different types of inflammatory insult to mediate temporally and spatially restricted signalling. ${ }^{1}$ This platelet recruitment in host defence and inflammation can occur in the absence of any overt vascular trauma or thrombus formation through 
mechanisms that are incompletely understood, but which are distinct from those required for haemostasis and thrombosis. ${ }^{1,18}$

Intriguingly, given the involvement of platelets in neutrophil recruitment into lung airspaces, platelet recruitment to lungs following inflammatory responses originating elsewhere in the body, such as LPSinduced peritonitis, ${ }^{19}$ or in the bloodstream following transfusion with anti-MHCl antibodies, ${ }^{20}$ has shown evidence of neutrophil dependence. Neutrophils have been identified as effector cells in mediating the responses to inhaled LPS, ${ }^{9,21}$ but the requirements for platelets in this context has not yet been explored. Understanding responses such as lung neutrophil and platelet recruitment, and how these are regulated may provide important information leading to new targets for the development of novel anti-inflammatory drugs.

The platelet adhesion molecules involved in the recruitment of platelets to lungs in host defence and inflammation have not yet been fully characterized. Potential candidates for this role are P-selectin, PSGL-1, a counter ligand of P-, E- and L-selectins, and ESL-1, a counter ligand to E-selectin. 3,5,8, 22-24 Study of these adhesion molecules offers potential identification of similarities and differences between platelet and neutrophil recruitment in inflammation.

We therefore developed methods for imaging lung platelet recruitment following LPS inhalation in mice, and then investigated the effects of blockade of P-selectin or PSGL-1, or the selective depletion of neutrophils, on this response.

\section{Materials and Methods}

Readers are directed to the online supplementary material for methodology related to: collection of mouse lungs for histology, immunohistochemistry using fluorescent reporters, immunohistochemistry using bright field DAB reporter, analysis of images of lung sections, flow cytometry analysis, 
experiments involving dabigatran etexilate, leukocyte and platelet counts from blood microsamples, and bronchoalveolar lavage (BAL) collection.

Mice

Mice (BALB/c strain, Female, 6-12 weeks of age) used at King's College London were obtained from Charles River (Harlow, UK) and housed in non-barrier facilities. Animal experiments were conducted according to ARRIVE guidelines, and the Animals (Scientific Procedures) Act, 1986, and 2012 amendments following local ethical approval. Terminal anaesthesia was achieved using urethane (3 $\mathrm{g} / \mathrm{kg}$, i.p.). Weight changes were monitored as an index of general health status (Supplementary Figure 1B), and it was noted that intervention studies outlined below (e.g blockade of PSGL-1, and Pselectin; and neutrophil depletion) had no effect on this parameter (Supplementary Figures $\mathbf{2} \mathbf{I}$ and 3E).

Experiments carried out at the University of California, San Francisco (UCSF) were approved by the institutional animal care and use committee. Mice were bred in specific pathogen-free barrier facilities at UCSF. Pf4-Cre $\times$ mTmG mice ${ }^{25}$ were generated from crosses of Pf4-Cre mice (C57BL/6-Tg(Pf4iCre)Q3Rsko/J, Jackson Laboratories), which express Cre recombinase largely restricted to the megakaryocyte and platelet lineage, ${ }^{26}$ with $\mathrm{mTmG}$ mice (Gt(ROSA)26Sortm4(ACTB-tdTomato,-EGFP)Luo/J, Jackson), in which a membrane targeted tandem dimer of tomato fluorescent protein (tdTomato, $\mathrm{mT}$ ) is constitutively globally expressed, unless Cre recombinase is expressed, where tdTomato gene expression is stopped and expression of membrane-targeted enhanced green fluorescent protein (eGFP, mG) is activated. ${ }^{27}$ Male mice at $8-12$ weeks of age were used for intravital microscopy experiments due to their larger size. Terminal anaesthesia was induced with $125 \mathrm{mg} / \mathrm{kg}$ ketamine/12.5 $\mathrm{mg} / \mathrm{kg}$ xylazine (i.p.). 


\section{Intranasal challenge with LPS}

To induce lung inflammation, mice were briefly anaesthetised with inhaled isoflurane, then challenged with LPS (from E. coli, O55:B5 serotype, Sigma Aldrich $5 \mathrm{mg} / \mathrm{kg}$ ), or PBS vehicle control, by intranasal delivery. Samples (lungs, BAL fluid) were collected 48 hours later as previously described. ${ }^{8,11}$

Administration of blocking or depleting antibodies and detection of circulating platelet-neutrophil complexes.

Neutrophil depletion was achieved with three doses of monoclonal anti-Ly6G antibody (clone 1A8, BioXCell, $25 \mathrm{mg} / \mathrm{kg}$ i.p.) at $-24,-1$ and +24 hours relative to intranasal challenge based on previously reported work. ${ }^{28}$ For experimental blockade of adhesion molecule function, either an anti-P-selectin monoclonal antibody (clone RB40.34, BD Biosciences), or an anti-PSGL-1 monoclonal antibody (clone 4RA10 BioXCell), was administered (4 mg/kg i.v. in PBS) at -1 and +24 hours relative to intranasal LPS challenge, with dose and frequency selected based on previously published work investigating the effects of LPS inhalation in mice, ${ }^{3}$ and other reports. ${ }^{14,29}$ Equivalent doses of monoclonal antitrinitrophenol (clone 2A3, BioXCell, trinitrophenol is not present in mammals) were administered to control mice. At 4 hours post challenge, some groups of mice were terminally anaesthetised and whole blood collected via cardiac puncture into citrated syringes for flow cytometric analysis of circulating platelet-neutrophil complexes.

\section{Intravital microscopy}

At 48 hours following PBS or LPS inhalation, Pf4-Cre $\times \mathrm{mTmG}$ mice were anaesthetised. The dorsal left ribcage was shaved, the trachea cannulated and the mouse attached to a Mini Vent ventilator with isoflurane and oxygen supplied at $130 \times 250 \mu \mathrm{l}$ breaths per minute with $2 \mathrm{cmH}_{2} \mathrm{O}$ of positive end expiratory pressure. Skin over the shaved region was removed, and an approximately $1 \mathrm{~cm}$ long intercostal incision was made to expose the ventilated left lung and allow the surface of the visceral 
pleura to fall away from the parietal pleura. Body temperature was maintained using a heated stage $\left(37^{\circ} \mathrm{C}\right)$ and mice administered PBS $(0.5 \mathrm{ml}$ i.p.) as fluid support.

As previously reported, ${ }^{25,30}$ a custom-made thoracic window was then inserted to sit between two ribs, secured onto a $90^{\circ}$ angle post clamp attached to the stage and 3-3.5 kPa negative pressure was applied between the visceral pleura of the lung and the inner surface of the window using an Amvex vacuum regulator in order to immobilise the lung, and to remove air from the pleural cavity.

After around 10 minutes of stabilisation, the lung was brought into focus on a custom Nikon A1R multiphoton microscope coupled with a MaiTai DeepSee infrared excitation laser tuned to $920 \mathrm{~nm}$ for simultaneous excitation of eGFP and tdTomato, using a x25 objective Nikon water immersion lens, and emission filters set for tdTomato at 570-620 nm and for eGFP at 500-550 nm. Image capture was controlled using NIS Element AR software with high resolution bi-directional Galvano scanning over $512 \times 512$ pixels.

The internal surface of the final generation of alveoli, a densely networked capillary bed, was brought into focus, and viability of the mouse confirmed by observation of blood flow in the pulmonary vessels. Then using a Z-drive the imaging plane was moved $10 \mu \mathrm{m}$ deeper into the lung to establish an imaging plane at a consistent depth into the lung. Video recordings at the maximum speed possible using Galvano scanning (0.91 frames per second) were then made in order to produce datasets where platelets adhesion in the lung microvasculature could be quantified. For each mouse, $8 \times$ videos of $230 \times 230 \mu \mathrm{m}$ fields with a duration of 5 minutes were sampled at different sites across the window, and videos were screened in a blinded fashion and excluded from analyses if stable imaging was not maintained, or if large blood vessels or megakaryocytes were in the sample region. 
Analysis of platelet adhesion using intravital image sequences

Intravital image sequences were analysed using NIS Elements 4.5 (Nikon) tracking. Platelets were identified by size and eGFP fluorescence intensity, and then platelet adhesion events were quantified by tracking platelets that did not move more than $2 \mu \mathrm{m} / \mathrm{s}$ between consecutive frames, whereas freeflowing or transiently adhesive platelets ('non-adhesive') were observed to appear and disappear between frames (Supplementary movie 1).

Experimental design and analysis.

For experiments using blocking or depleting antibody treatment interventions, the experimenter was blinded and randomization within cage blocks was used to assign mice into treatment groups. Other experiments were carried out unblinded, with blinding applied for analysis of samples or images.

Comparisons between two independent groups were made using two-tailed unpaired Student's ttests. Correlations were analysed using Pearson's test to derive the $\mathrm{R}^{2}$ value describing goodness of fit, with an F-test for the probability that $R^{2}$ values from experimental data would be generated from randomly generated datasets with no correlation. Where appropriate, single or repeated measures 1way analysis of variance (ANOVA), 2-way ANOVA, 3-way ANOVA, or three way ANCOVA with baseline values fit as covariates were used.

P-values reported are from t-tests, F-tests on Pearson's correlations, or multiplicity-adjusted P-values (Dunnett's or Holm's adjustments where appropriate). The threshold for statistical significance was set at $\mathrm{P}<0.05$.

Data are graphed and reported as means \pm standard error. Group sizes $(\mathrm{n})$ are listed in figure legends. Excel 2016 (Microsoft Office), InVivoStat 3.6 (InVivoStat), and Prism 6.0 (Graphpad). 


\section{Results}

Platelets and neutrophils are recruited to the lungs after LPS administration in mice.

To record platelet and neutrophil recruitment in the same lung tissue sections of mice administered intranasal LPS, we undertook histological analysis of frozen lung sections stained for CD41 (integrin $\alpha$ Ilb, platelet marker), and neutrophil elastase using immunofluorescence (Figures 1A \& 1B). Quantitative analysis of tissue sections revealed a significant increase in pulmonary platelet recruitment in mice administered LPS compared to PBS-administered control mice (Figure 1C), as well as an increase in lung neutrophil elastase staining (Figure 1D). Platelets in lung sections appeared larger with LPS inhalation, although the majority of platelets in sampled fields resembled single platelet events and did not display the morphology of megakaryocytes or thrombi (Supplementary figure 1A).

LPS inhalation was previously shown to decrease blood platelet counts, ${ }^{8}$ and LPS challenge in the present study also decreased blood platelet counts at 4 hours after challenge, but this thrombocytopaenia was transient, returning to levels observed in control mice at 24 hours after challenge (Figure $\mathbf{1 H}$ ), providing evidence that changes in platelet numbers in lung sections containing blood at 48 hours post LPS were due to platelet recruitment to lungs rather than changes in numbers of circulating blood platelets.

Immunofluorescence staining allowed for identification of spatial associations of platelets with neutrophil elastase within lung sections (Figure 1E). LPS inhalation increased the quantity of platelets both associated with, and not associated with neutrophil elastase in the lungs (Figure 1F), with an increase in the percentage of platelets that were neutrophil elastase-associated (Figure 1G). Interventional studies were therefore carried out to further investigate whether interactions of platelets with neutrophils were a causative factor in LPS-induced lung platelet recruitment. 
P-selectin and PSGL-1 are dispensable for LPS-induced lung platelet recruitment

Previous observations of the increased presence of neutrophil-associated platelets in the lungs after LPS administration in lung sections (Figures $\mathbf{1 F}, \mathbf{1 G} \& \mathbf{1 H}$ ), and using intravital lung imaging, ${ }^{8,11}$ and the requirement of platelets and contact-dependent PSGL-1 interactions for LPS-induced pulmonary neutrophil recruitment, ${ }^{3}$ led us to speculate that platelet recruitment might be dependent on the formation of platelet-neutrophil conjugates mediated through PSGL-1. A bright field method was developed for higher-throughput quantification of the platelet marker CD42b (GP1b $\alpha$, Figure 2A, as well as for neutrophil elastase, Supplementary Figure 1C), and the involvement of P-selectin and PSGL-1 in LPS-induced lung platelet recruitment was tested by administering functional blocking antibodies to mice (Figure 2B).

As reported previously, ${ }^{3}$ the administration of an anti-PSGL-1 blocking antibody led to significant and selective reduction in pulmonary neutrophil recruitment to lung airspaces (Figure 2C, Supplementary Figures 2A \& 2B). Indeed, 4 hours after LPS exposure, the percentage of events that are neutrophils attached to platelets in the circulating leukocyte population was significantly increased in LPS compared to PBS administered mice $(P<0.001$. Figure 2D), The administration of an anti-PSGL-1 blocking antibody or anti-P-selectin blocking antibody led to a significant reduction in the percentage of events that are neutrophils attached to platelets in the circulating leukocyte population attached to platelets $(P<0.05$. Figure $2 \mathrm{D})$. In contrast, the presence of platelets in the lungs was not reduced in mice administered either an anti-P-selectin, or anti-PSGL-1 blocking antibody (Figures 2E \& 2F). Together, these data suggest that platelet recruitment to lungs following LPS inhalation occurs via a distinct mechanism compared to the mechanisms required for neutrophil recruitment, which show dependence on PSGL-1.

Additionally, neither P-selectin nor PSGL-1 blockade affected the decrease in blood platelet counts at 4 hours following LPS inhalation (Figure 2G), whilst PSGL-1 blockade decreased blood mononuclear cell counts at 4 hours, and blood neutrophil counts at 48 hours; P-selectin blockade increased blood neutrophil counts 48 hours following LPS inhalation (Supplementary Figures 2C-E). The latter effect is 
a potential mechanism for increases in neutrophil elastase staining in lung sections with anti-P-selectin antibody treatment (Supplementary Figures 2 G \& $2 \mathrm{H}$ ).

LPS-induced platelet recruitment to lungs is not affected by neutrophil depletion.

To investigate whether pulmonary platelet recruitment was dependent on neutrophils, mice were depleted of circulating neutrophils using an anti-Ly6G antibody (Figure 3A). Anti-Ly6G antibody administration depleted neutrophils from circulating blood in both PBS control mice and in LPSchallenged mice in which inflammation drives greatly enhanced neutrophil production following LPS inhalation over the entire 48 hours (Figure 3B \& 3E). The anti-Ly6G antibody treatment also reduced neutrophil elastase staining in both control and LPS-challenge lungs (Figures 3C \& 3D), and reduced neutrophil recruitment to lung airspaces (Figure 3E), with monocyte recruitment to lung airspaces unaffected (Supplementary Figures 3A \& 3B). Circulating blood mononuclear cells were not affected by the administration of anti-Ly-6G antibody, apart from 4 hours after LPS administration (Supplementary figure 3C \& 3D), possibly due to the involvement of mononuclear cells in antibodymediated neutrophil clearance. ${ }^{31}$

The depletion of neutrophils had no detected effect on lung platelet recruitment (Figures 4A \& 4B). Furthermore, LPS administration resulted in an increase in the platelet granule protein PF4 in BAL supernatant, which was not affected by the depletion of neutrophils (Figure 4C). The immunodepletion of neutrophils resulted in a reduction in circulating blood platelet counts, irrespective of, and additional to effects of, LPS administration, at 4 hours, 24 hours and 48 hours (Figure 4D), which could be anticipated to bias towards a finding of decreased lung platelet recruitment in lung sections containing blood. There was a complete absence of CD42b events (platelets) in the lungs of mice depleted of platelets using an anti-platelet depleting antibody, or using the staining protocol in the absence of the primary detection antibody, to signify the robustness of the imaging system and 
quantification methods, in the absence of a PSGL-1, P-selectin, or neutrophil dependent effect (Supplementary Figure 4).

We also hypothesised that if platelet recruitment was dependent on neutrophil recruitment, mice with high LPS-induced neutrophil recruitment to lungs would have high LPS-induced lung platelet recruitment. Lung platelet (CD42b), and neutrophil elastase staining quantifications from serial sections from all LPS-treated mice were combined (pooled from control mice data in Figures 2E, 3D, 4B and 8 additional mice, data shown in Supplementary Figures 5A \& 5B, shown together in Figures 4F \& 4G), and tested for correlation. No significant correlation between extent of neutrophil and platelet recruitment was observed (Figure $\mathbf{4 H}$ ). Additionally, the extent of lung platelet (CD41) and neutrophil elastase staining showed no evidence of any correlation (Supplementary figure 5C, from data in Figures 1C \& 1D), again providing no evidence supportive of neutrophil dependence of LPSinduced platelet recruitment.

Imaging LPS-induced platelet recruitment in living lungs reveals increased platelet adhesion in the lung microvasculature without platelet aggregation.

In order to further understand the nature of the interactions of platelets with the pulmonary vasculature, and to directly image the effects of LPS inhalation on platelets in the living lung microcirculation, two photon intravital microscopy video recordings were made in lungs of Pf4-Cre $x$ mTmG mice at 48 hours after intranasal exposure to LPS or PBS. The effect of LPS inhalation on the quantity of lung platelet-leukocyte interactions imaged using intravital microscopy has been previously explored using simultaneous genetic labelling of platelets and leukocytes in mice, ${ }^{11}$ but total platelet recruitment has not previously been measured. Two photon intravital microscopy in Pf4Cre $\times \mathrm{mTmG}$ mice, reporter mice with high eGFP fluorophore density on platelet membranes, enabled improved imaging of single platelets passing through the lung microvasculature (Figure 5A), as previously reported. ${ }^{25}$ Image sequences were captured at 0.91 frames per second for 5 minutes from 
several fields across the visible lung, and tracking software was used to isolate adhesive platelets, which did not move faster that $2 \mu \mathrm{m} / \mathrm{s}$ over 3 or more frames, from platelets which were free-flowing in the bloodstream that appeared and disappeared with each frame (Figure 5B, Supplementary movie 1). LPS inhalation increased the number of adhesive platelets detected in lungs, and no time effect was detected, indicating stable levels of platelet adhesion during acquisition of images (Figures $\mathbf{5 C} \mathbf{\&}$ 5D). The mean duration of platelet adhesion events increased, although not significantly after LPS inhalation (Figure 5E). The imaged microvasculature received blood flow during imaging, and nonadhesive platelets were observed in quantities that did not differ between mice challenged with LPS or PBS controls (Figures $\mathbf{5 F} \boldsymbol{\&} \mathbf{5 G}$ ). It was confirmed that mice used for imaging had raised inflammatory responses to LPS (Figure $5 \mathbf{H}$ ).

Live imaging revealed that platelet recruitment to the lungs appeared to require vascular adhesion, and platelets did not appear to be attached to neutrophils, although in the microvasculature leukocytes were not easily discriminated from other Pf4- tdTomato+ cells in the lung. Furthermore, platelets appeared as single platelets or small aggregates, and no gross platelet aggregation was evident in the lung microvasculature, and the LPS-induced platelet adhesion events were visually distinct from thromboemboli induced by intravenous infusion of $100 \mathrm{lU} / \mathrm{kg}$ human $\alpha$-thrombin (Enzyme Research Laboratories) (Figure 5I, Supplementary movie 2), or haemostatic thrombosis occurring as a result of incision into the lung surface during intravital window insertion (Figure $\mathbf{5 J}$, Supplementary movie 3). Furthermore the administration of the direct thrombin inhibitor dabigatran etexilate, at a dose sufficient to significantly increase tail bleeding time (Supplementary Figure 6A), and suppress ex vivo platelet aggregation towards thrombin from pooled platelet rich plasma (Supplementary Figure 6B), did not suppress platelet recruitment to the lungs in response to LPS (PBS + vehicle : $320.1 \pm 180.0$ platelets $/ \mathrm{mm}^{2}$; LPS + vehicle: $806.6 \pm 314.1$ platelets $/ \mathrm{mm}^{2} ;$ LPS + dabigatran: $757.7 \pm 490.2$ platelets $/ \mathrm{mm}^{2}$ Supplementary Figure 6C. Data not significantly different between groups) or event size (Supplementary Figure 6D. Data not significantly different between groups), 
suggesting that platelet adhesion was not likely to be dependent on fibrin deposition in the lungs as a result of thrombin activation.

As previously reported, ${ }^{25}$ occasional megakaryocyte thrombopoeisis events were observed which had an appearance distinct from adhesive platelets, with these large cells captured in small arterioles and moving over minutes to extend proplatelet projections (Figure 5K, Supplementary movie 4), although sample regions and durations required for platelet adhesion tracking were insufficient for quantification of megakaryocyte thrombopoiesis.

\section{Discussion}

This is the first report investigating the relationship of neutrophils with lung platelet recruitment in response to bacterial LPS administered via inhalation. We show that despite the close association of recruited platelets with neutrophils in the lungs, ultimately, platelet recruitment showed no dependence on the co-recruitment of neutrophils, nor P-selectin or PSGL-1 mediated interactions classically associated with platelet-dependent neutrophil recruitment. It is possible that platelet interaction with the vascular endothelium after LPS administration is dependent on other adhesive mechanisms that are attributable to platelet adhesion to damaged vessel walls during trauma, for example platelet GPIb interacting with vWF, or platelet GPIIb/IIla binding to fibrinogen on the vessel wall. ${ }^{32-34}$ However, the administration of a direct thrombin inhibitor (dabigatran etexilate) in this LPS model did not affect platelet recruitment, suggestive that fibrin was not an adhesive substrate for platelets under these conditions. Furthermore, the fact that the bleeding time is not different between mice administered LPS compared to PBS suggests that disseminated intravascular coagulation does not occur in this model. 
The relationship between platelets and neutrophils during the innate immune response is complex and involves multiple processes. An overriding theme of this relationship is the requirement for platelets to increase the efficiency of neutrophil recruitment to areas of inflammation or infection. Selectin, and integrin-mediated events provide both an increase in platelet-neutrophil circulating complexes in blood, ${ }^{3}$ increased neutrophil rolling and adhesion events on the endothelial surface, ${ }^{12}$ increased neutrophil intravascular crawling, ${ }^{14}$ and increased neutrophil diapedesis into tissue. ${ }^{15}$ Furthermore, platelets are sometimes found associated with extravascular neutrophils. ${ }^{11,13}$ Interestingly, although no evidence was found in the present study for requirement of neutrophils of pulmonary platelet recruitment, we still observed a highly significant increase in the spatial association of platelets with neutrophils in the lungs after LPS administration. Platelets influence neutrophil effector processes in a contact-dependent manner, for example free-radical formation and phagocytosis, ${ }^{35}$ transcellular metabolism of pro-inflammatory lipids, ${ }^{36}$ and involvement in NET formation. ${ }^{37-39}$ It is not known if these neutrophil activation processes occur due to a different population of platelets accessing lung tissue that are not initially associated with intravascular neutrophil recruitment events, but this is something worthy of follow up experiments.

These findings contrast with previous reports studying the effects of anti-Gr1 antibody mediated neutrophil depletion on lung recruitment of radiolabelled platelets following intraperitoneal injections of LPS, ${ }^{19}$ or following priming with intraperitoneal administration of LPS, followed by intravenous anti$\mathrm{MHCl}$ antibody administration in a model of transfusion-related acute lung injury (TRALI). ${ }^{20}$ This may be explained by differences between anti-Ly6G (1A8) and anti-Gr1 (RB6-8C5) antibodies, with the latter depleting Ly6C+ inflammatory monocytes in addition to Ly6G+ neutrophils, ${ }^{28}$ a difference which can have functional consequences bacterial infection models. ${ }^{40}$ In addition, different routes of LPS administration can produce differing inflammatory responses. For example, in 'organ-on-a-chip' models, platelet recruitment to human lung endothelial cells requires epithelial rather than endothelial exposure to LPS, ${ }^{41}$ intraperitoneal LPS administration promotes lung neutrophil recruitment as a distant organ affect away from the inflammatory focus in the peritoneum, and the 2- 
hit model of TRALI where LPS acts as a priming agent involves a rapid intravascular response in the lung in which injury is not dependent on PSGL-1. ${ }^{20}$ Furthermore, unlike previous reports, we were unable to detect pulmonary platelet recruitment at early time points ( 4 hours following administration of LPS, data not shown). ${ }^{19}$ However, platelet sequestration might be occurring in different tissues at this earlier time point in our model, made evident by thrombocytopaenia at 4 hours post LPS administration.

Intravital imaging experiments in living lungs provide further evidence that platelet activation and recruitment in inflammation contrasts with that in haemostasis and thrombosis, ${ }^{18,42}$ as platelet adhesion to the pulmonary vascular endothelium occurred as single events in the absence of platelet aggregation. Intravital microscopy experiments have recently demonstrated a novel immune function of platelet adhesion in which platelets migrate on endothelial cells in the infected liver endothelium in order to accumulate fibrinogen-bound bacteria, with this intravascular crawling of lone platelets shown to still occur following neutrophil and monocyte depletion with anti-Gr-1. ${ }^{43}$ However, the efficiency of this process in these circumstances is difficult to determine. ${ }^{43}$ The convoluted 3 dimensional anatomy of the lung capillary network, lung movements during ventilation, and the sterile LPS model prevented measurements of directed platelet migration in the lungs from imaging reported in the present study, but the platelet adhesion observed in lungs following LPS inhalation is indicative that a similar platelet migration response may occur in the inflamed lung. Indeed, inhalation of LPS, ${ }^{11}$ bacteria, ${ }^{13}$ and other inflammatory insults, ${ }^{44}$ can promote migration of platelets into airspaces, and platelets are important for limiting lung infection by Klebsiella pneumoniae, ${ }^{45}$ Streptococcus pneumoniae ${ }^{46}$ and Pseudomonas aeruginosa. ${ }^{13}$ The distinct recruitment of platelets from other recruited inflammatory cells might suggest a role for platelets in the inflammatory response in their own right. Whilst it is not yet evident whether direct platelet microbicidal activity is important for host defence, platelets do contain potent microbicidal activity both in vitro and in vivo. ${ }^{39,47}$ 
Interventional studies reported here revealed that neutrophil depletion and subsequent LPS inhalation did not cause shock and mortality as reported with intravenous LPS or TNF- $\alpha$ priming followed by intravenous anti-Gr-1 (RB6-8C5) or anti-Ly6G (1A8) antibodies. ${ }^{48,49}$ However, modest decreases in body weight and platelet counts in anti-Ly6G-treated mice in both PBS and LPS-treated mice suggested that some milder adverse effects were present, and were additional to LPS effects. It is possible that these effects in LPS-challenged mice relate to a requirement for neutrophil myeloperoxidase to limit inflammation following exposure to high doses of LPS. 50

The findings that blockade of P-selectin increased, whilst PSGL-1 blockade decreased, blood neutrophil counts in the context of lung inflammation are of note for several reasons. First, they are concordant with knockout mouse studies in which genetic deletion of P-selectin increased steady-state blood neutrophil counts, ${ }^{51}$ but contrast with data from PSGL-1 knockout mice in which baseline neutrophil counts are elevated. ${ }^{52}$ Second, these findings implicate P-selectin and PSGL-1 as potential molecular mediators of blood neutrophil homeostasis under pressure from acute inflammation. Finally, it is possible that anti-PSGL-1 antibodies which have been characterised as having a blocking function, may potentially deplete circulating neutrophils during inflammation. ${ }^{14,53}$

To conclude, we report for the first time the lack of neutrophil-dependence of platelet recruitment in lung inflammation following LPS inhalation, a model where lung airspaces are the inflammatory focus and neutrophils are the major cellular infiltrate. The lung platelet recruitment did not depend on Pselectin or PSGL-1-mediated adhesive interactions and involved platelet adhesion in the absence of platelet aggregation. These findings provide further support for a role for platelets in host defence and inflammation in the lungs that is mechanistically separate from neutrophil recruitment, and distinct from platelet adhesion in thrombosis and haemostasis.

Acknowledgements. We wish to thank Abhinav Kumar and Val Millar for guidance with imaging at the Stevenage Bioscience Catalyst, and Kyle Marchuk and Adam Fries for support with imaging at the Biological Imaging Development Centre, UCSF. 


\section{Figure legends}

Figure 1. LPS inhalation causes platelet and neutrophil recruitment to the lungs, increased spatial associations of platelets in lungs with neutrophil elastase, and a transient decrease in blood platelet counts. (A) Mice were administered PBS or LPS (5mg/kg) intranasally, and lungs collected at 48 hours. (B) Platelet CD41 and neutrophil elastase were stained in frozen lung sections using immunofluorescence. CD41+ platelets are in red, neutrophil elastase staining is in green and Hoechststained nuclei are in blue. (C) Quantification of number of CD41+ platelets per $\mathrm{mm}^{2}$ lung tissue; and (D) neutrophil elastase staining. (E) Identification of visibly neutrophil elastase-associated platelets and platelets not associated with neutrophils elastase, with output from Cell Profiler shown below input images (neutrophil elastase-associated platelets recolored in magenta, with overlap in white, highlighted with white circles). (F) Quantification of platelets not visibly spatially associated, and those spatially associated with neutrophil elastase, and $(G)$ percentage of platelets visibly spatially associated with neutrophil elastase. $(\mathrm{H})$ Blood platelet counts from serial tail blood microsamples. Scale bars $=20 \mu \mathrm{m}$. Mean \pm standard error, Imaging: PBS group $n=6$, LPS group $n=7$, blood counts: both $\mathrm{n}=8$, unpaired t-test or 2 way ANOVA with repeated measures and Holm's test for LPS effects, Flow cytometric analysis was conducted with $n=4$ per group, and analysed using one way ANOVA with Dunett's test between groups, ${ }^{*}=\mathrm{P}<0.05,{ }^{* *}=\mathrm{P}<0.01,{ }^{* * *}=\mathrm{P}<0.001$.

Figure 2. PSGL-1 blockade suppresses LPS-induced lung neutrophil recruitment but does not affect LPS-induced lung platelet recruitment. (A) Bright field staining of platelet CD42b (brown, black in quantification output in bottom row) with nuclei counterstain (htox, blue). (B) Mice were administered either control IgG, anti-P-selectin blocking antibody (RB40.34), or anti-PSGL-1 blocking 
antibody (4RA10) at $4 \mathrm{mg} / \mathrm{kg}$ i.v. $-1 \mathrm{~h}$, and $+24 \mathrm{~h}$ in relation to intranasal administration of PBS or LPS. (C) BAL neutrophil counts. (D) In some mice, a cardiac puncture performed 4h post LPS administration to measure via flow cytometric analysis the percentage of leukocytes identified to be neutrophils bound to platelets (E) Platelet CD42b immunostaining with CD42b DAB reporter positivity (brown in top row, with haematoxylin counterstain in blue) and deconvolution of DAB signal black in bottom row, from same images as top row) for image analysis. Scale bars $=20 \mu \mathrm{m}$. (F) Quantification of CD42b+ platelets in lung tissue. (G) Blood platelet counts from serial tail microsamples. Means \pm standard error, (F, G) PBS group: $n=4$, LPS groups: $n=8$; (C) PBS group: $n=8$, LPS groups $n=16$. (D) PBS group: $n=4$, LPS groups n=4. (C, D, F) One-way ANOVA, or (G) two-way ANOVA, with Dunnett's or Holm's test for difference vs. LPS+control IgG group, ${ }^{*}=\mathrm{P}<0.05,{ }^{* *}=\mathrm{P}<0.01,{ }^{* * *}=\mathrm{P}<0.001$.

Figure 3. Treatment with anti-Ly6G antibody depletes neutrophils in blood and lungs. (A) Mice were administered either control IgG or anti-Ly6G antibody at $-24 h,-1 h$, and $+24 h$ in relation to intranasal administration of PBS or LPS (5mg/kg). (B) Serial tail blood microsamples were used to quantify blood neutrophils immediately before (0h), and at $+4 \mathrm{~h},+24 \mathrm{~h}$, and $+48 \mathrm{~h}$ after PBS or LPS administration (note break in axis). (C) Neutrophil elastase was immunostained in lung sections, and in sample fields neutrophil elastase DAB reporter positivity (brown in top row) was deconvoluted (black in bottom row, from same images as top row) for image analysis. Scale bar $=20 \mu \mathrm{m}$. (D) Quantification of neutrophil elastase in lung sections. (E) Other groups of mice were sacrificed at 48 hours and BAL neutrophils were quantified. Means \pm standard error, $(A) n=12,(D, E) n=6$. Data analysed via: (A) 3way ANOVA with repeated measures and Holm's test for LPS and anti-Ly6G treatment effects within time points, or (D, E) 2-way ANOVA with Holm's test for LPS and anti-Ly6G treatment effects, $* *=\mathrm{P}<0.01, * * *=\mathrm{P}<0.001$. 
Figure 4. LPS-induced lung platelet recruitment is unaffected by neutrophil depletion. Mice were administered either control IgG or anti-Ly6G antibody at $-24 h,-1 h$, and $+24 h$ in relation to intranasal administration of PBS or LPS (5mg/kg). (A) Platelet CD42b was immunostained in lung sections, and in sample fields CD42b DAB reporter positivity (brown in top row) was deconvoluted (black in bottom row, from same images as top row) for image analysis. Scale bar $=20 \mu \mathrm{m}$. (B) Quantification of platelets in lung sections. (C) Separate groups of mice were sacrificed at 48 hours after intranasal challenge for BAL for the measurement of platelet factor 4 (PF4) via ELISA. (D) Blood platelet counts from serial tail blood microsamples. (F) CD42b+ lung platelet counts and (G) extent of lung neutrophil elastase staining data pooled from all PBS and LPS-challenged control mice and additional mice (Supplementary figures 4A \& 4B) were used for correlation measurements of extent of neutrophil elastase staining vs. lung platelet staining from mice administered with LPS with line of best fit and residuals. One neutrophil elastase reading of $21.65 \%$ coverage (Figure 3D) is not shown in (G) for scaling reasons but was included in analyses, and exclusion did not affect conclusion of analysis). Means \pm standard error, $(B, C) n=6$ or $(D, E) n=12$. Analysis was $(B, C)$ 2-way ANOVA, or (D) 3-way ANOVA with repeated measures, all followed by Holm's test for LPS and anti-Ly6G treatment effects, $(F, G)$ unpaired t-test and $(H)$ Pearson's correlation, $\mathrm{R}^{2}$ and result of F-test for significantly non-zero slope shown, ${ }^{*}=\mathrm{P}<0.05,{ }^{* *}=\mathrm{P}<0.01, * * *=\mathrm{P}<0.001$.

Figure 5. LPS inhalation increases platelet adhesion in lungs which is distinguishable from lung haemostatic thrombosis and thromboembolism. (A) Pf4-Cre $\times$ mTmG mice were administered PBS or LPS $(5 \mathrm{mg} / \mathrm{kg})$ intranasally, and 48 hours later were prepared for two-photon lung intravital microscopy to image tdTomato+ lung cells (red) and eGFP+ platelets (green). (B) Diagram showing the identification of adhesive platelets (blue) compared to the temporary sightings of platelets free flowing in blood (green) over 3 seconds, with white dotted lines demonstrating between-frame platelet tracking (see Supplementary movie 1). (C) Quantification of adhesion events over 300 second sample image sequences, and (D) mean summary data showing data from each video and mean data 
from each mouse used for statistical comparisons. (E) mean adhesive platelet adhesion duration data from each field of lung sampled with means from each mouse. (F) Quantification of platelet events which were not tracked between frames over time (either freely flowing in blood or making interactions too transient for tracking), and (G) mean summary data from each field of lung sampled with means from each mouse. $(H)$ Neutrophil counts in BAL from mice used in intravital experiments, sampled after completion of imaging. (I) Pulmonary thromboembolism induced by injection of 100 IU/kg of thrombin i.v. during imaging. (J) Appearance of haemostatic platelet thrombi 20 minutes after incision into lung surface (highlighted with white dashed line, see Supplementary movie 2). (K) Appearance of megakaryocyte trapping and thrombopoeisis in the lung (highlighted with white line, see Supplementary movie 3). Scale bars $=20 \mu \mathrm{m}$. Means \pm standard error, $n=4,2$-way ANOVA with repeated measures or unpaired t-tests, ${ }^{*}=\mathrm{P}<0.05,{ }^{* * *}=\mathrm{P}<0.001$.

\section{References}

1. Middleton EA, Weyrich AS, Zimmerman GA. Platelets in Pulmonary Immune Responses and Inflammatory Lung Diseases. Physiol. Rev. 2016;96(4):1211-59.

2. Kolaczkowska E, Kubes P. Neutrophil recruitment and function in health and inflammation. Nat. Rev. Immunol. 2013;13(3):159-75.

3. Kornerup KN, Salmon GP, Pitchford SC, Liu WL, Page CP. Circulating platelet-neutrophil complexes are important for subsequent neutrophil activation and migration. J. Appl. Physiol. 2010;109(3):758-767.

4. Page $\mathrm{C}$, Pitchford S. Neutrophil and platelet complexes and their relevance to neutrophil recruitment and activation. Int. Immunopharmacol. 2013;17(4):1176-84.

5. Hidalgo A, Chang J, Jang J-E, et al. Heterotypic interactions enabled by polarized neutrophil microdomains mediate thromboinflammatory injury. Nat. Med. 2009;15(4):384-91.

6. Amison RT, Momi S, Morris A, et al. RhoA signaling through platelet $\mathrm{P} 2 \mathrm{Y}_{1}$ receptor controls leukocyte recruitment in allergic mice. J. Allergy Clin. Immunol. 2015;135(2):528-38.

7. Amison RT, Arnold S, O'Shaughnessy BG, et al. Lipopolysaccharide (LPS) induced pulmonary neutrophil recruitment and platelet activation is mediated via the $\mathrm{P} 2 \mathrm{Y} 1$ and $\mathrm{P} 2 \mathrm{Y} 14$ receptors in mice. Pulm. Pharmacol. Ther. 2017;45:62-68.

8. Pan D, Amison RT, Riffo-vasquez Y, et al. P-Rex and Vav Rac-GEFs in platelets control leukocyte recruitment to sites of in fl ammation. Blood. 2015;125(7):1146-1159. 
9. Grommes J, Alard JE, Drechsler M, et al. Disruption of platelet-derived chemokine heteromers prevents neutrophil extravasation in acute lung injury. Am. J. Respir. Crit. Care Med. 2012;185(6):628-636.

10. Duerschmied D, Suidan GL, Demers M, et al. Platelet serotonin promotes the recruitment of neutrophils to sites of acute inflammation in mice. Blood . 2013;121(6):1008-1015.

11. Ortiz-Muñoz G, Mallavia B, Bins A, et al. Aspirin-triggered 15-epi-lipoxin A4 regulates neutrophil-platelet aggregation and attenuates acute lung injury in mice. Blood. 2014;124(17):2625-34.

12. Wang HB, Wang JT, Zhang $L$, et al. P-selectin primes leukocyte integrin activation during inflammation. Nat. Immunol. 2007;8(8):882-892.

13. Amison RT, O'Shaughnessy BG, Arnold S, et al. Platelet Depletion Impairs Host Defence to Pulmonary Infection with Pseudomonas aeruginosa in Mice. Am. J. Respir. Cell Mol. Biol. 2017;rcmb.2017-00830C.

14. Sreeramkumar V, Adrover JM, Ballesteros I, et al. Neutrophils scan for activated platelets to initiate inflammation. Science. 2014;346(6214):1234-8.

15. Zuchtriegel G, Uhl B, Puhr-Westerheide D, et al. Platelets Guide Leukocytes to Their Sites of Extravasation. PLOS Biol. 2016;14(5):e1002459.

16. Ley K, Laudanna C, Cybulsky MI, Nourshargh S. Getting to the site of inflammation: the leukocyte adhesion cascade updated. Nat. Rev. Immunol. 2007;7(9):678-689.

17. Semple JW, Italiano JE, Freedman J. Platelets and the immune continuum. Nat. Rev. Immunol. 2011;11(4):264-274.

18. Page CP. The involvement of platelets in non-thrombotic processes. Trends Pharmacol. Sci. $1988 ; 9(2): 66-71$.

19. Andonegui G, Kerfoot SM, McNagny K, et al. Platelets express functional Toll-like receptor-4. Blood. 2005;106(7):2417-2423.

20. Looney MR, Nguyen JX, Hu Y, et al. Platelet depletion and aspirin treatment protect mice in a two-event model of transfusion-related acute lung injury. J. Clin. Invest. 2009;119(11):3450.

21. Savov JD, Gavett SH, Brass DM, Costa DL, Schwartz DA. Neutrophils play a critical role in development of LPS-induced airway disease. 2002;283(5):L952-L962.

22. Carlow DA, Gossens K, Naus S, et al. PSGL-1 function in immunity and steady state homeostasis. Immunol. Rev. 2009;230(1):75-96.

23. Frenette PS, Denis C V, Weiss L, et al. P-Selectin glycoprotein ligand 1 (PSGL-1) is expressed on platelets and can mediate platelet-endothelial interactions in vivo. J. Exp. Med. 2000;191(8):1413-1422.

24. Pitchford SC, Momi S, Giannini S, et al. Platelet P-selectin is required for pulmonary eosinophil and lymphocyte recruitment in a murine model of allergic inflammation. Blood. 2005;105(5):2074-2081.

25. Lefrançais E, Ortiz-Muñoz G, Caudrillier A, et al. The lung is a site of platelet biogenesis and a reservoir for haematopoietic progenitors. Nature. 2017;544(7648):105-109.

26. Tiedt R, Schomber T, Hao-Shen H, Skoda RC. Pf4-Cre transgenic mice allow the generation of lineage-restricted gene knockouts for studying megakaryocyte and platelet function in vivo. 
Blood. 2007;109(4):1503-6.

27. Muzumdar MD, Tasic B, Miyamichi K, Li L, Luo L. A global double-fluorescent Cre reporter mouse. genesis. 2007;45(9):593-605.

28. Daley JM, Thomay AA, Connolly MD, Reichner JS, Albina JE. Use of Ly6G-specific monoclonal antibody to deplete neutrophils in mice. J. Leukoc. Biol. 2008;83(1):64-70.

29. Phillips JW, Barringhaus KG, Sanders JM, et al. Single injection of P-selectin or P-selectin glycoprotein ligand-1 monoclonal antibody blocks neointima formation after arterial injury in apolipoprotein E-deficient mice. Circulation. 2003;107(17):2244-2249.

30. Looney MR, Thornton EE, Sen D, et al. Stabilized imaging of immune surveillance in the mouse lung. Nat. Methods. 2011;8(1):91-6.

31. Bruhn KW, Dekitani K, Nielsen TB, Pantapalangkoor P, Spellberg B. Ly6G-mediated depletion of neutrophils is dependent on macrophages. Results Immunol. 2016;6:5-7.

32. Jackson SP, Mistry N, Yuan Y. Platelets and the injured vessel wall-'rolling into action'. Trends Cardiovasc. Med. 2001; 10: 192-197.

33. Wei AH, Schoenwaelder SM, Andrews RK, Jackson SP. New insights into the haemostatic function of platelets. Br. J. Haematol. 2009; 147: 415-430.

34. Vargo-Szabo D, Pleines I, Nieswandt B. Cell adhesion mechanisms in platelets. Arterioscler. thromb. Vasc. Biol. 2008; 28:403-412

35. Peters MJ, Dixon G, Kotowicz KT, et al. Circulating platelet-neutrophil complexes represent a subpopulation of activated neutrophils primed for adhesion, phagocytosis and intracellular killing. Br. J. Haematol. 1999;106(2):391-9.

36. Maugeri N, Evangelista V, Celardo A, et al. Polymorphonuclear leukocyte-platelet interaction: role of P-selectin in thromboxane B2 and leukotriene C4 cooperative synthesis. Thromb. Haemost. 1994;72(3):450-6.

37. Clark SR, Ma AC, Tavener SA, et al. Platelet TLR4 activates neutrophil extracellular traps to ensnare bacteria in septic blood. Nat. Med. 2007;13(4):463-469.

38. Caudrillier A, Kessenbrock K, Gilliss BM, et al. Platelets induce neutrophil extracellular traps in transfusion-related acute lung injury. J. Clin. Invest. 2012;122(7):2661-71.

39. Kraemer BF, Campbell RA, Schwertz H, et al. Novel Anti-bacterial Activities of $\beta$-defensin 1 in Human Platelets: Suppression of Pathogen Growth and Signaling of Neutrophil Extracellular Trap Formation. PLoS Pathog. 2011;7(11):e1002355.

40. Shi C, Hohl TM, Leiner I, et al. Ly6G+ neutrophils are dispensable for defense against systemic Listeria monocytogenes infection. J. Immunol. 2011;187(10):5293-8.

41. Jain A, Barrile R, van der Meer AD, et al. A primary human lung alveolus-on-a-chip model of intravascular thrombosis for assessment of therapeutics. Clin. Pharmacol. Ther. 2017;

42. Pitchford SC. Novel uses for anti-platelet agents as anti-inflammatory drugs. Br. J. Pharmacol. 2007;152(7):987-1002.

43. Gaertner F, Ahmad Z, Rosenberger G, et al. Migrating Platelets Are Mechano-scavengers that Collect and Bundle Bacteria. Cell. 2017;171(6):1368-1382.e23.

44. Pitchford SC, Momi S, Baglioni S, et al. Allergen induces the migration of platelets to lung tissue in allergic asthma. Am. J. Respir. Crit. Care Med. 2008;177(6):604-612. 
45. de Stoppelaar SF, van 't Veer C, Claushuis TAM, et al. Thrombocytopenia impairs host defense in gram-negative pneumonia derived sepsis. Blood. 2014;

46. van den Boogaard FE, Schouten M, de Stoppelaar SF, et al. Thrombocytopenia Impairs Host Defense During Murine Streptococcus pneumoniae Pneumonia. Crit. Care Med. 2015;43(3):.

47. Yeaman MR. Platelets: at the nexus of antimicrobial defence. Nat Rev Micro. 2014;12(6):426437.

48. Abbitt KB, Cotter MJ, Ridger VC, et al. Antibody ligation of murine Ly-6G induces neutropenia, blood flow cessation, and death via complement-dependent and independent mechanisms. J. Leukoc. Biol. 2009;85(1):55-63.

49. Tanaka Y, Nagai Y, Kuroishi T, Endo Y, Sugawara S. Stimulation of Ly-6G on neutrophils in LPSprimed mice induces platelet-activating factor (PAF)-mediated anaphylaxis-like shock. J. Leukoc. Biol. 2011;91(3):485-494.

50. Reber LL, Gillis CM, StarkI P, et al. Neutrophil myeloperoxidase diminishes the toxic effects and mortality induced by lipopolysaccharide. J. Exp. Med. 2017;

51. Robinson SD, Frenette PS, Rayburn H, et al. Multiple, targeted deficiencies in selectins reveal a predominant role for P-selectin in leukocyte recruitment. Proc. NatI. Acad. Sci. U. S. A. 1999;96(20):11452-7.

52. Xia L, Sperandio $M$, Yago $T$, et al. P-selectin glycoprotein ligand-1-deficient mice have impaired leukocyte tethering to E-selectin under flow. J. Clin. Invest. 2002;109(7):939-50.

53. Miner JJ, Xia L, Yago T, et al. Separable requirements for cytoplasmic domain of PSGL-1 in leukocyte rolling and signaling under flow. Blood. 2008;112(5):2035-2045. 

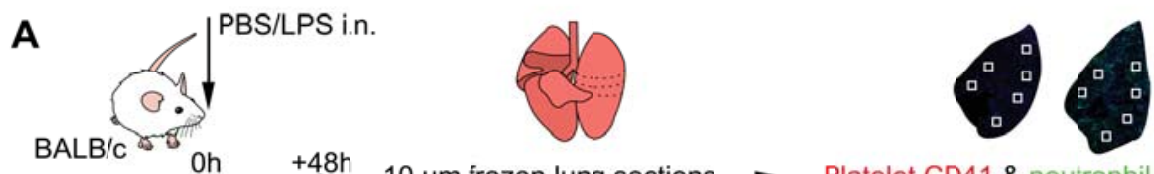

$\longrightarrow 10 \mu \mathrm{m}$ frozen lung sections $\rightarrow$

Platelet CD41 \& neutrophil elastase IHC
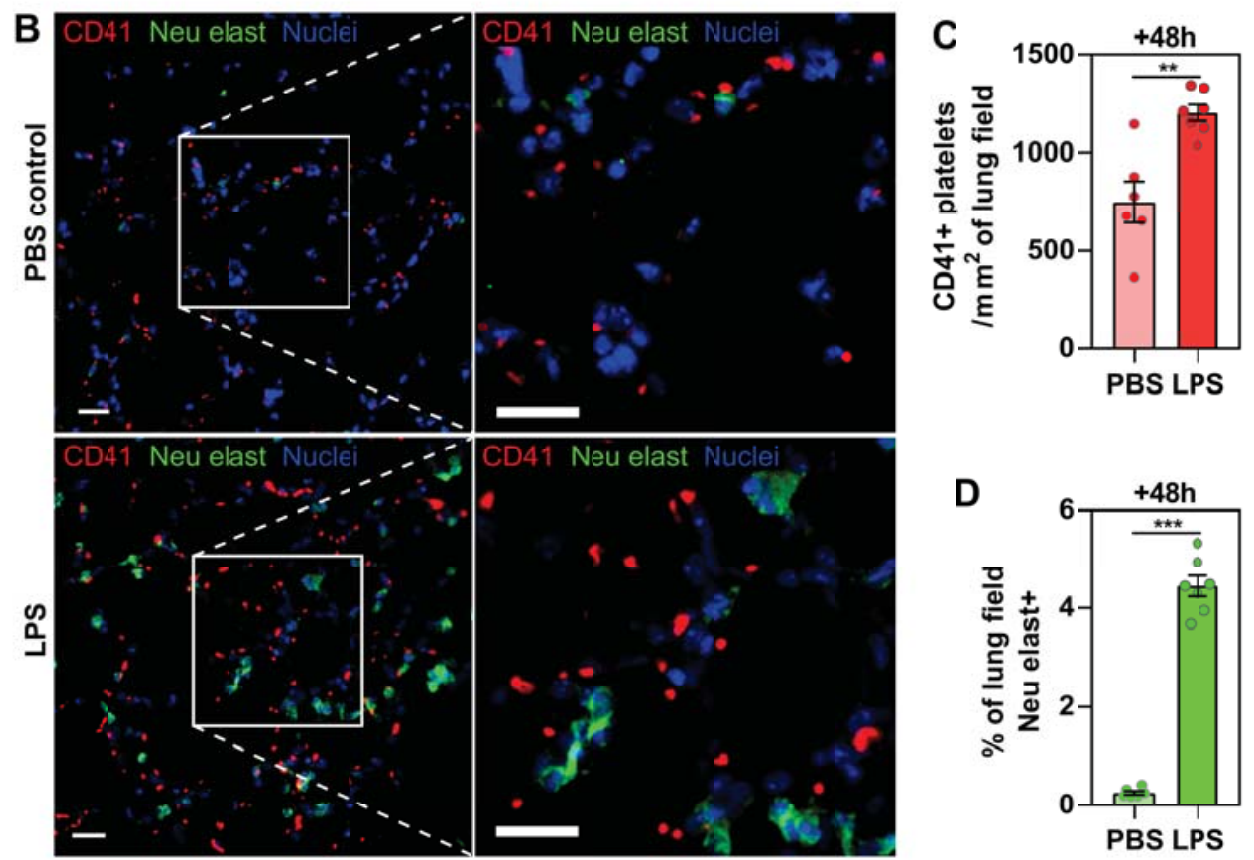
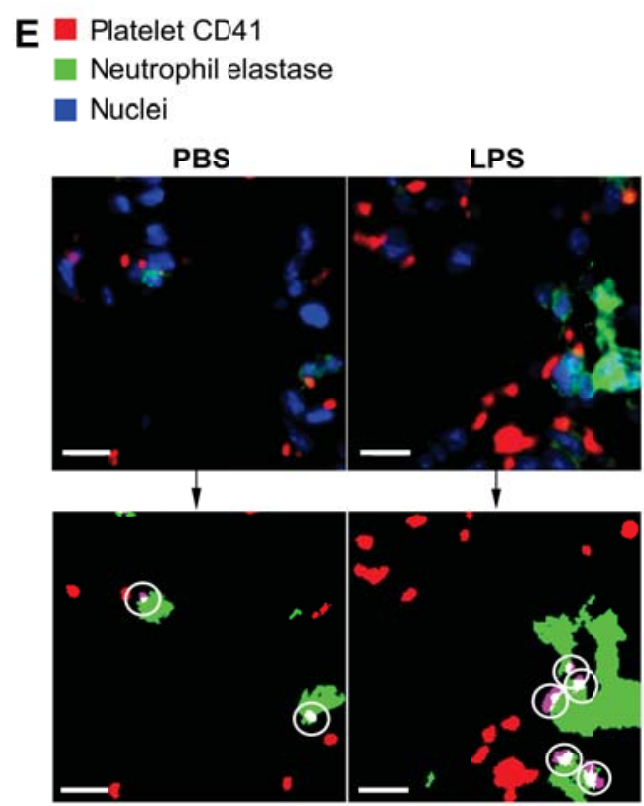

Platelets (assoc. $w /$ neu elast)

Platelets (not assoc. w/ neu elast)

$\square$ Neu elast
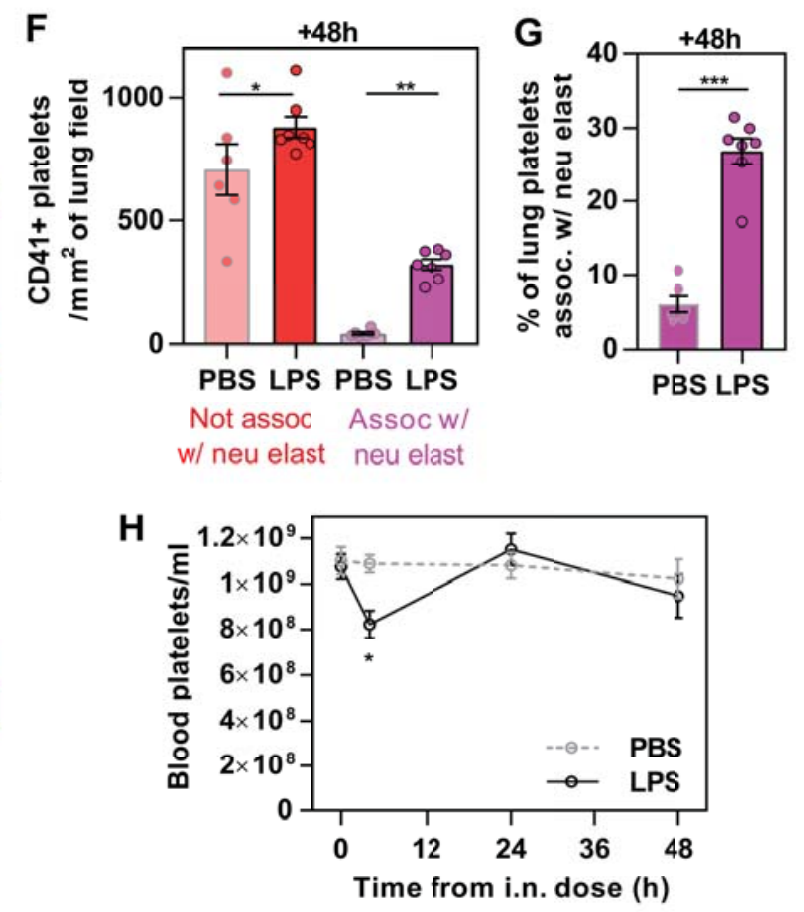

Figure 1 
A

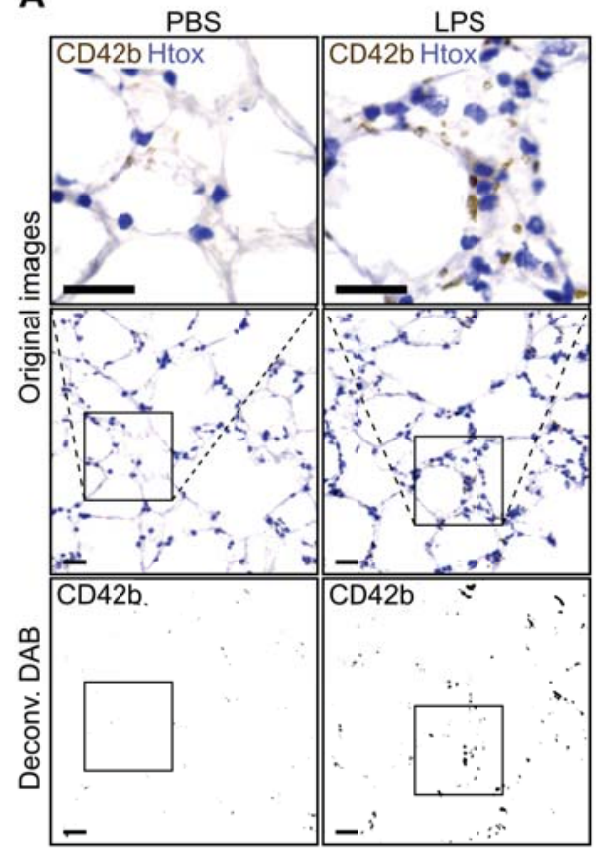

B

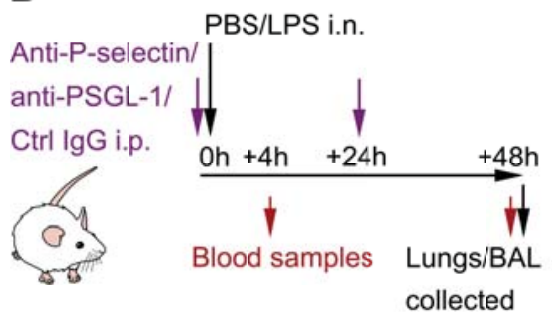

C
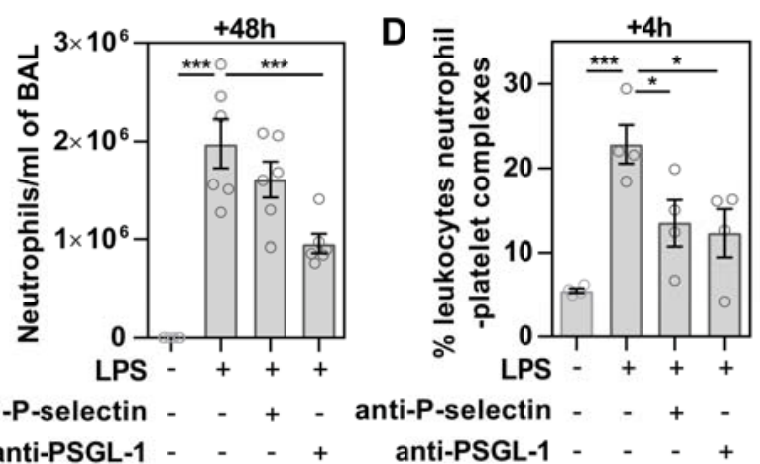

E

LPS
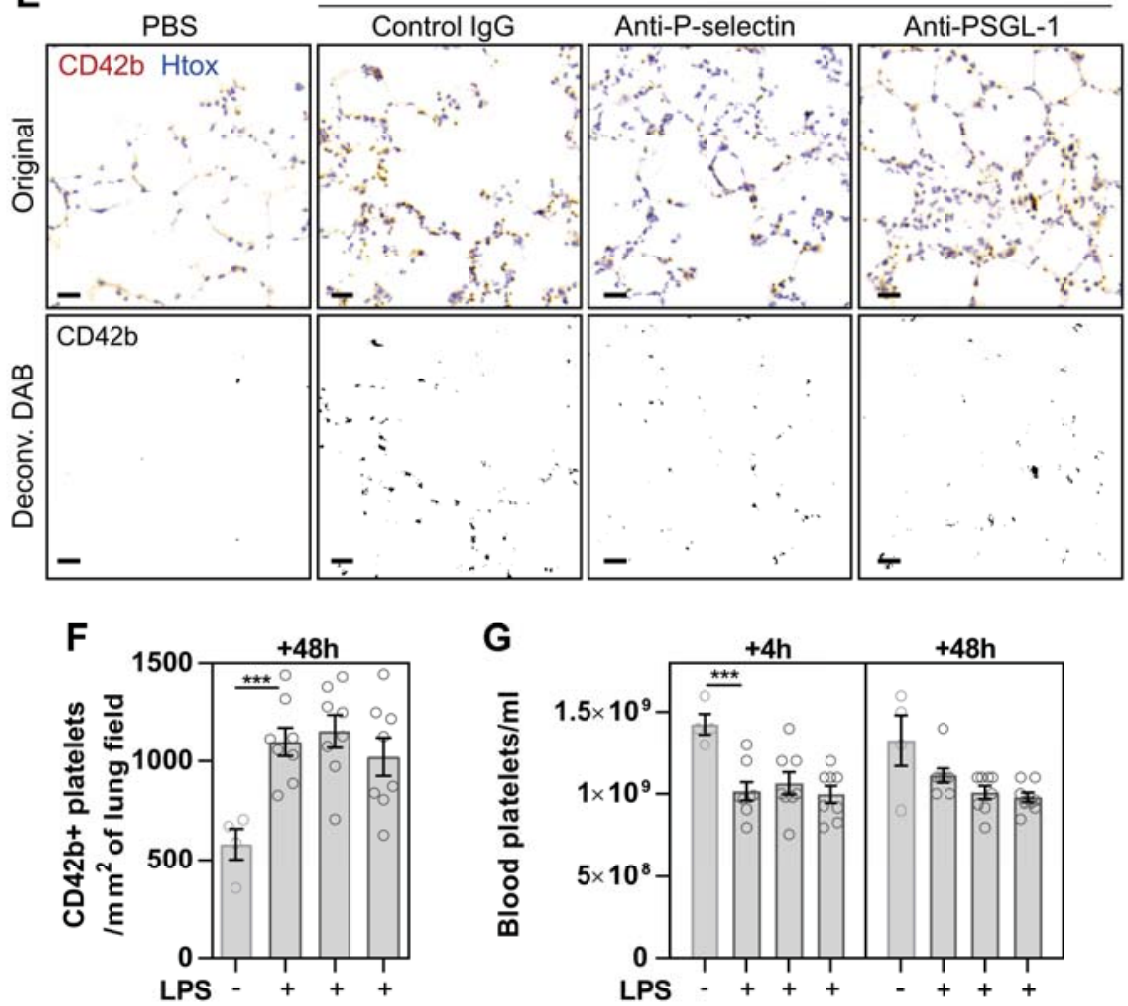

anti-P-selectin - - + -

anti-P-selectin - - + - - - + -

anti-PSGL-1 - - - +

anti-PSGL-1 - - - + - - - +

Figure 2 

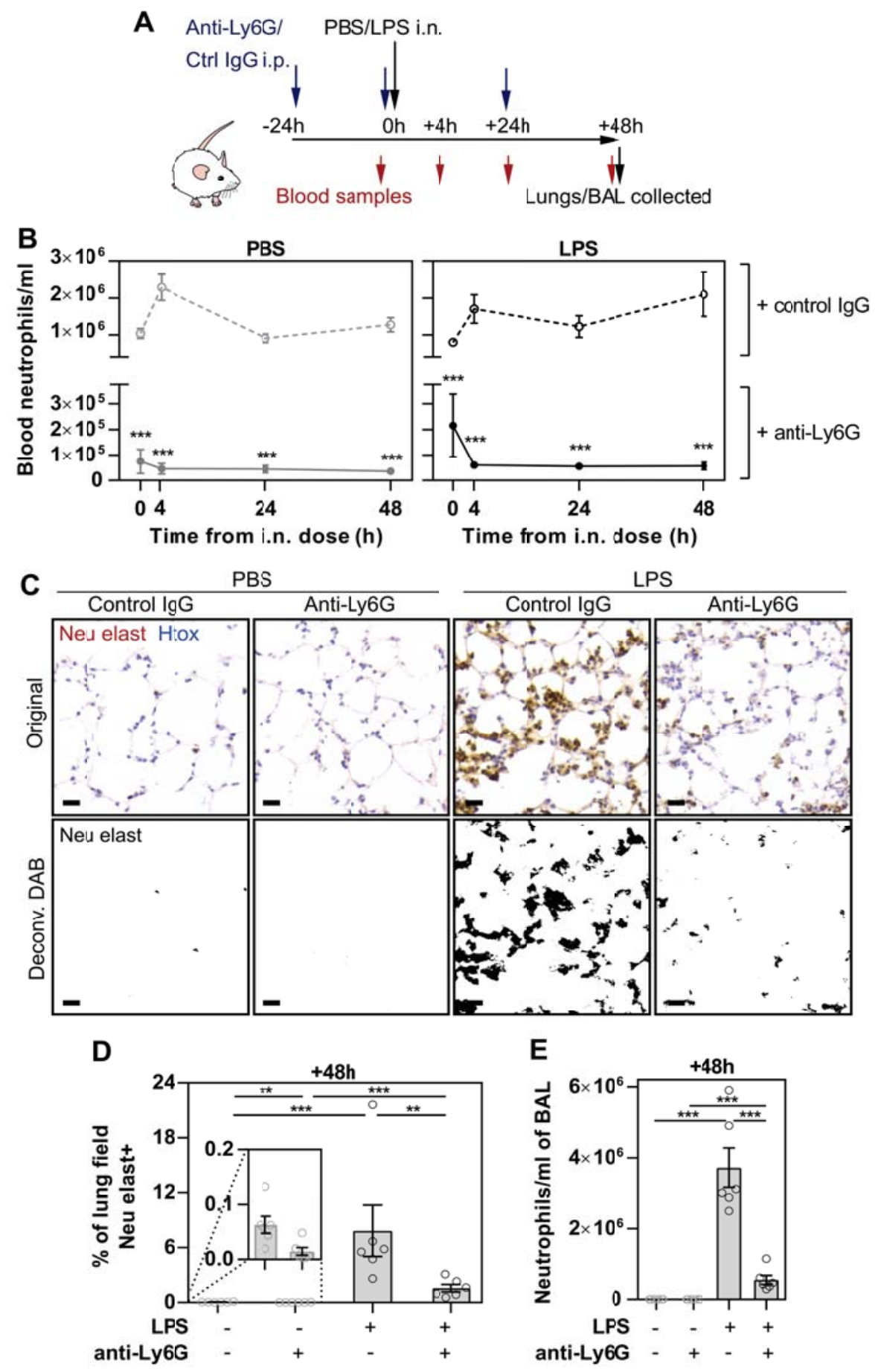

Figure 3 
A

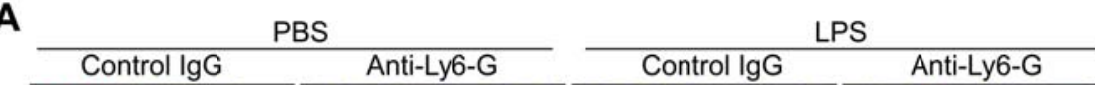
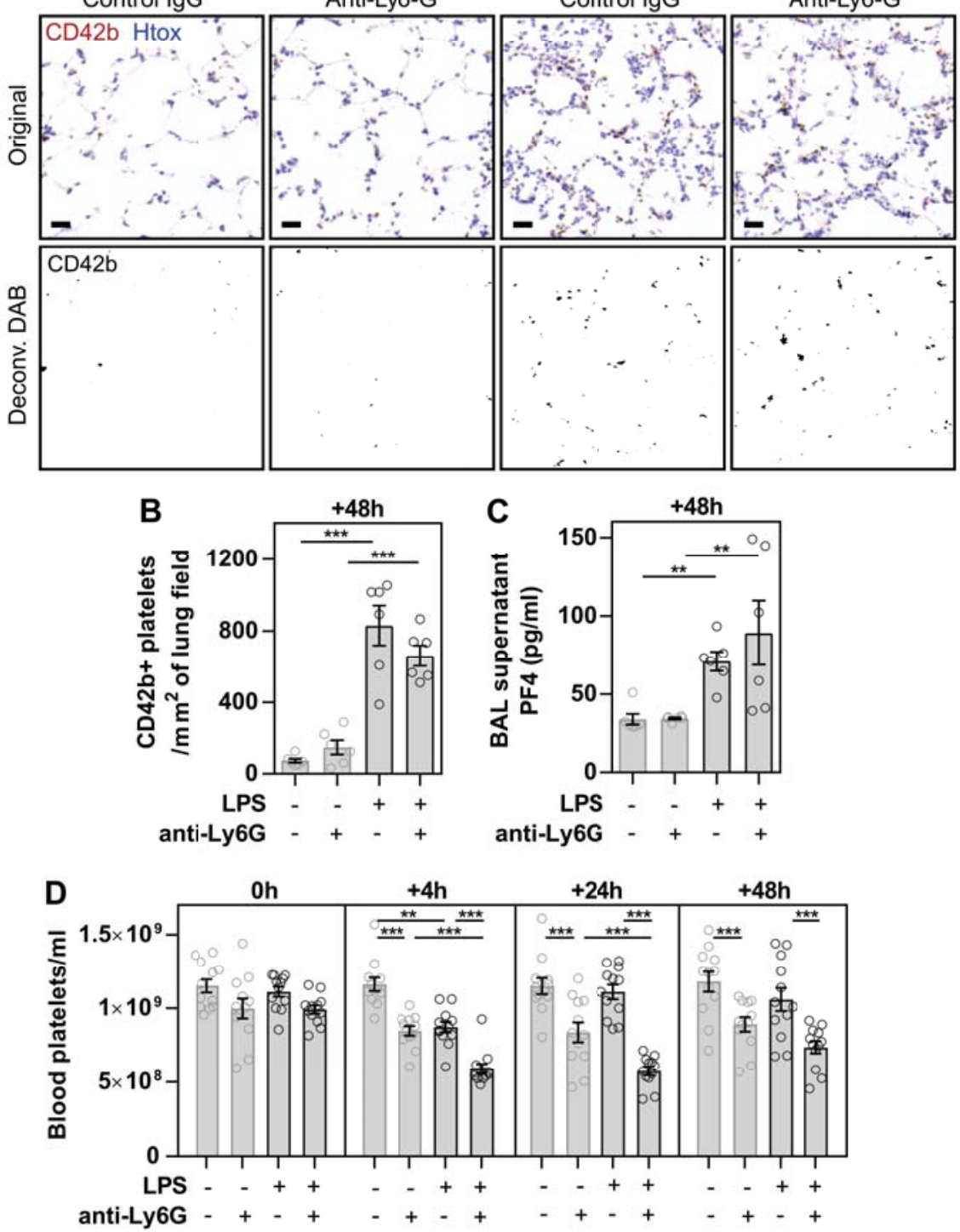

Pooled data from control mice

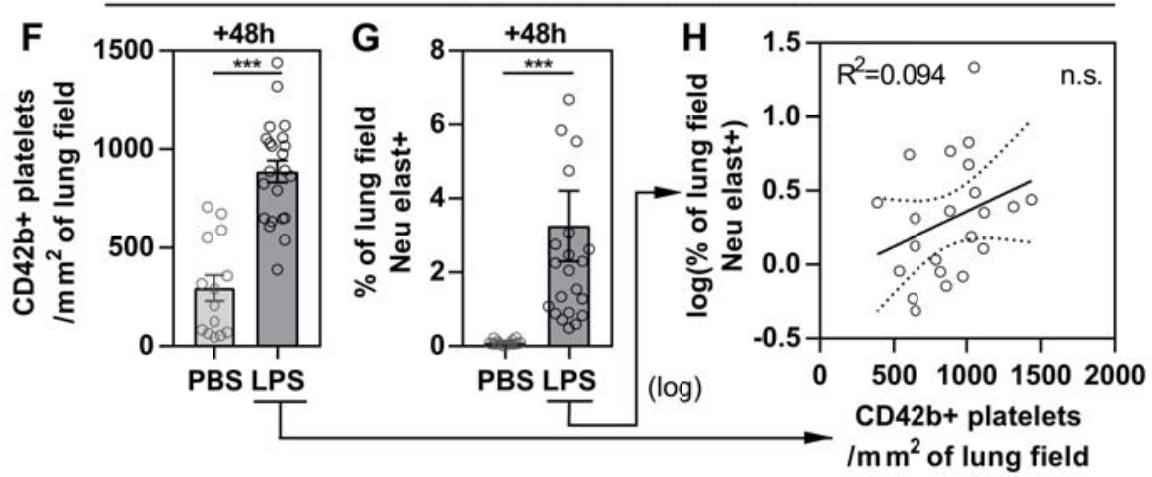

Figure 4 


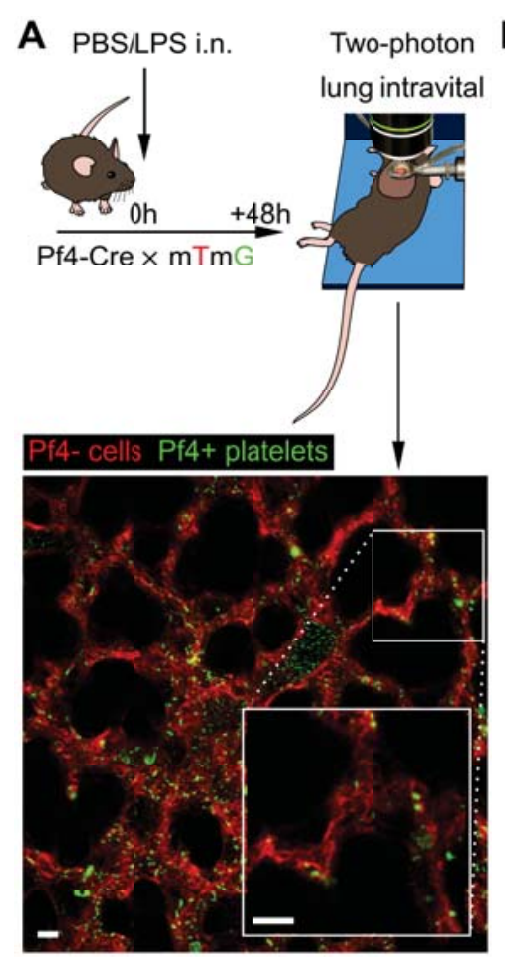

B a Pf4- cells

Platelets (not adhesive)

- Platelets (adhesive)

Platelet adhesion tracking

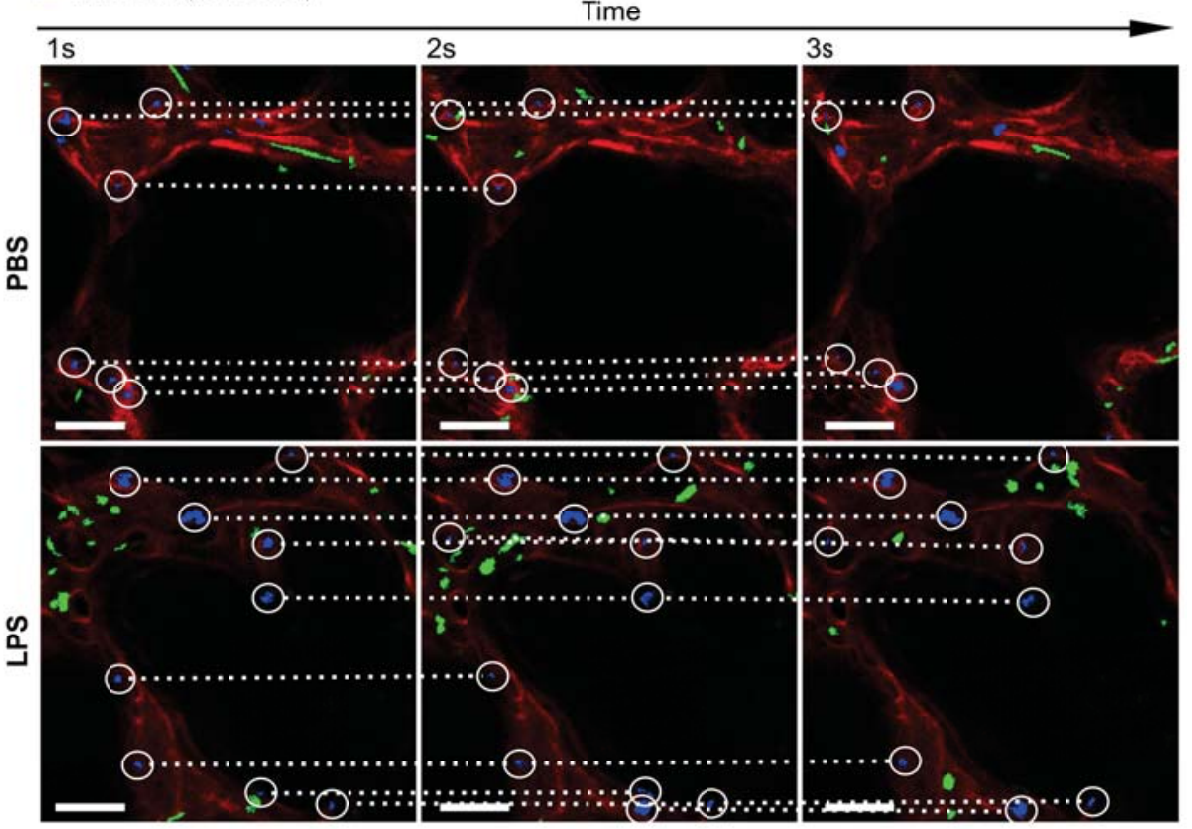

C
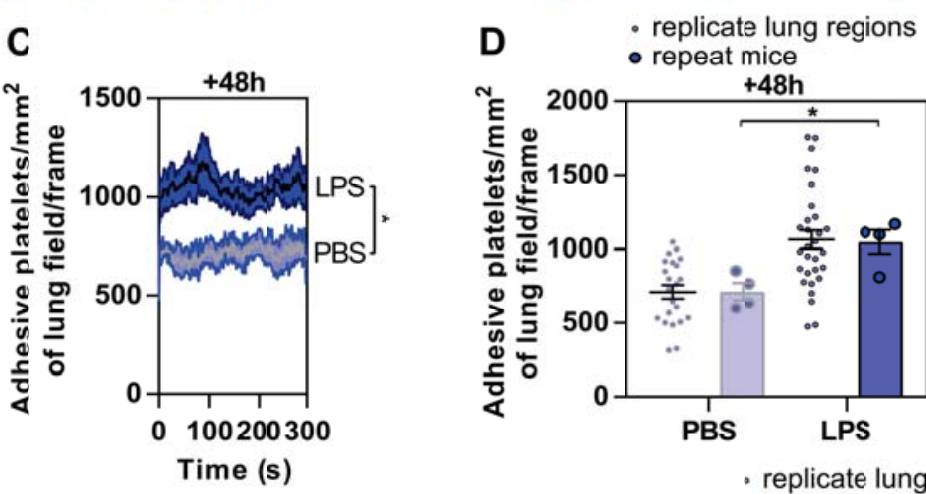

E replicate lung regions
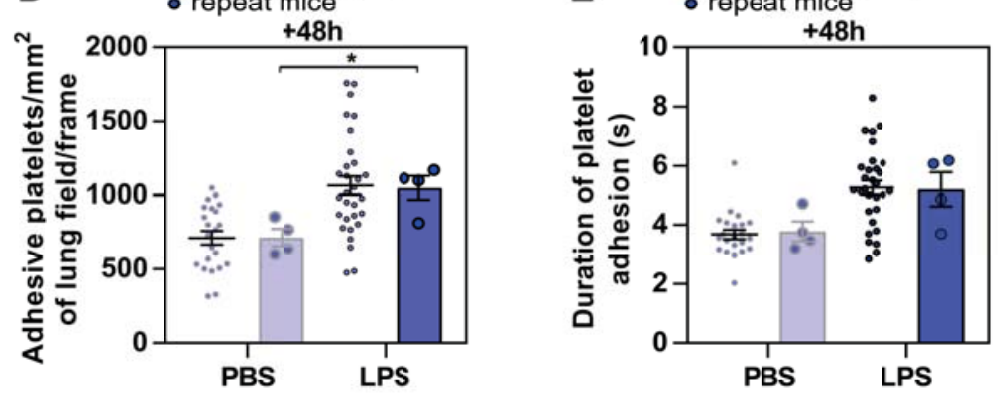

$\mathbf{F}$
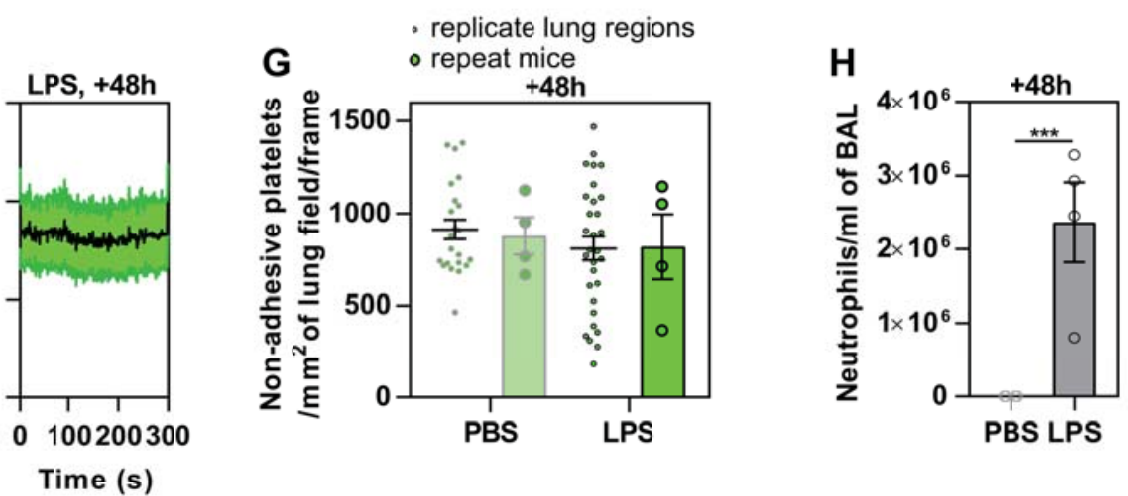

I Thromboembolism (100 IU/kg thrombin i.v.)

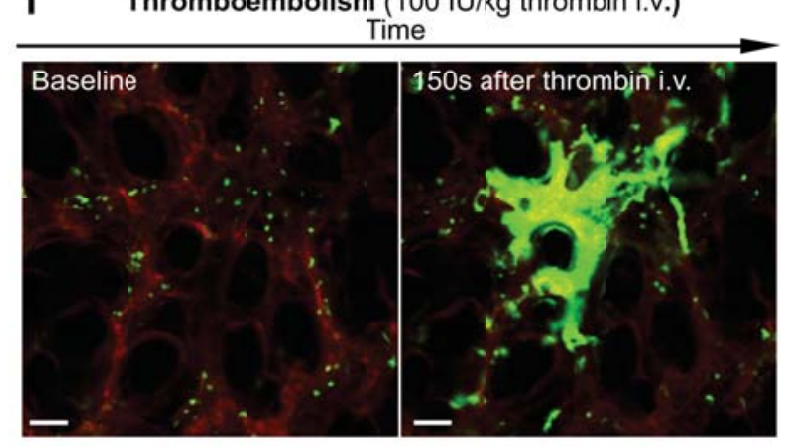

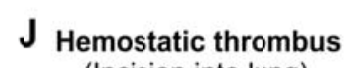
(Incision into lung)

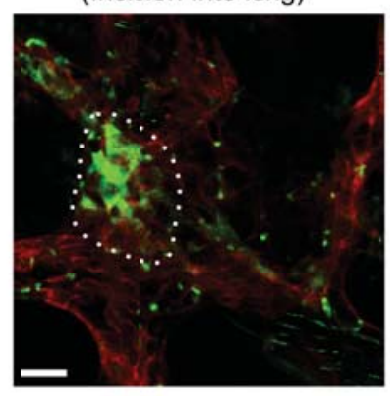

K

K Megakaryocyte

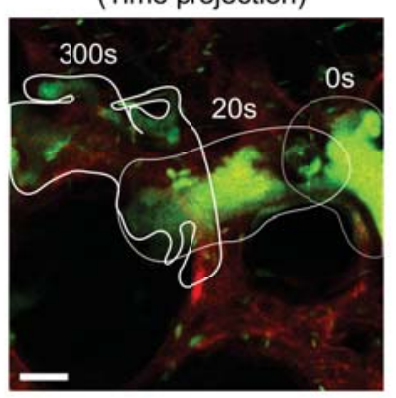

\section{Figure 5}




\section{ONLINE SUPPLEMENTARY MATERIAL}

\section{LPS-induced lung platelet recruitment occurs independently from neutrophils, PSGL-1, and P-selectin.}

Simon J. Cleary, ${ }^{1}$ Carl Hobbs, ${ }^{2}$ Richard T. Amison, ${ }^{1}$ Stephanie Arnold, ${ }^{1}$ Blaze G. O'Shaughnessy, ${ }^{1}$ Emma Lefrançais, ${ }^{3}$ Beñat Mallavia, ${ }^{3}$ Mark R. Looney, ${ }^{3}$ Clive P. Page, ${ }^{1}$ Simon C. Pitchford. ${ }^{1 *}$

${ }^{1}$ Sackler Institute of Pulmonary Pharmacology, Institute of Pharmaceutical Science, King's College London, London, UK.

${ }^{2}$ The Wolfson Centre for Age Related Diseases, King's College London, London, UK.

${ }^{3}$ University of California San Francisco (UCSF), Department of Medicine, San Francisco, USA

\section{Methods}

Collection of mouse lungs for histology

Mice were terminally anaesthetised, the trachea was opened and the diaphragm dissected along the ventral ribcage to collapse the lungs. A tracheal cannula was introduced and the lungs were inflated with $0.5 \mathrm{ml}$ of OCT (optical cutting temperature compound, VWR International). The heart was then excised to stop circulation, the left lung lobe was removed, cross-sectioned in half laterally, placed cut face down in plastic $15 \times 15 \mathrm{~mm}$ moulds (VWR International), and immersed with OCT. Samples were then snap frozen in liquid nitrogen and subsequently stored at $-80^{\circ} \mathrm{C}$ until required for use. Frozen $10 \mu \mathrm{m}$ cross-sections of tissue (6-9 per mouse) were made using a Model OT cryostat (Bright Instruments) and thaw-mounted onto Superfrost+ slides (VWR International).

Immunohistochemistry using fluorescent reporters.

For immunofluorescence experiments, dried sections were fixed for 15 minutes in $4 \%$ paraformaldehyde in PBS, tap water washed, then non-specific binding blocked for 10 minutes using 1\% BSA (Fraction V, Sigma Aldrich) in PBS, which was also used as diluent for later reagents. After a tap water wash, rat anti-mouse CD41 primary antibody (clone MWReg30, Abcam) and rabbit anti-mouse/human neutrophil elastase primary antibody (polyclonal ab68672, Abcam) were co-incubated with the sample for 2 hours. Following a PBS wash, anti-mouse IgG conjugated to AlexaFluor 594 (Thermo Fisher Scientific), anti-rabbit IgG conjugated to AlexaFluor 488 (Thermo Fisher Scientific) and Hoechst 33258 DNA stain (Sigma Aldrich) were incubated together on the sample in 1\% BSA in PBS for 1 hour before a final PBS wash. Slides were mounted in Fluoromount $\mathrm{G}$ (Southern Biotech) and stored in the dark at $4^{\circ} \mathrm{C}$ overnight before imaging. 
Fluorescence micrographs were captured using an InCell Analyser 6000 system (GE Healthcare) with a 20× objective, open aperture, excitation/emission wavelength filter sets for AlexaFluor 594: 561/605nm, AlexaFluor 488: 488/525nm and Hoechst 33258: 405/455nm. Image fields were $665.6 \times 665.6 \mu \mathrm{m}$. Tiled acquisition was used with 15\% overlap for stitching and both IR laser autofocus on the slide/section interface, and software autofocus on the Hoechst channel to maintain focus whilst tiling across the entire section.

Immunohistochemistry using bright field DAB reporter.

Immunofluorescence allowed for the simultaneous visualisation of neutrophil elastase, but required the use of an antirat secondary antibody reporter to amplify CD41 immunostaining, which introduced interference in cell depletion and adhesion molecule blocking experiments where mice were dosed in vivo with antibodies raised in rats. A method was developed using CD42b (GP1ba platelet marker) using a new monoclonal antibody raised in rabbits and 3,3'diaminobenzidine (DAB) reporters allowing bright field imaging.

Dried frozen sections of lung tissue were immersed in $4 \% \mathrm{vol} / \mathrm{vol}$ paraformaldehyde (Sigma Aldrich) in PBS and fixed for 15 minutes. Following a tap water wash, endogenous peroxidase activity was blocked and endogenous pigment bleached by immersion in $3 \% \mathrm{vol} / \mathrm{vol}$ hydrogen peroxide in ethanol (Sigma Aldrich). Slides were then blocked as above and anti-CD42b (SP219 clone, Abcam) or anti-neutrophil elastase antibodies were incubated with the sample for 2 hours. After a tap water wash samples were then incubated with biotinylated anti-rabbit IgG antibodies (BA-1000, Vector labs) for 1 hour, washed again and incubated with avidin and biotinylated horseradish peroxidase complex (Vectastain Elite, Vector labs) for 1 hour before a final wash and development with $1.2 \mathrm{mM}$ DAB (Sigma Aldrich) in 0.1 M Tris buffer with $0.03 \%$ hydrogen peroxide for 10 minutes, then counterstaining with Gill's no. 2 hematoxylin (Sigma Aldrich) and mounting in DPX.

With 6 transverse sections of middle left lung collected per mouse, using a 20x objective on a DM 2000 LED bright field microscope with DFC295 camera (Leica) and Leica Application Suite acquisition software $v 4.4,6 \times$ fields of $447 \times 596 \mu \mathrm{m}$ of the respiratory portion of lung were captured across each section.

\section{Analysis of images of lung sections}

Images were analyzed using ImageJ 1.48 (National Institutes of Health). Sample fields consisting of $6 \times 666 \times 666 \mu \mathrm{m}$ regions of fluorescent images were chosen in a blinded fashion based on the nuclei channel. The colour deconvolution 
plugin produced by G. Landini, University of Birmingham (Colour deconvolution: http://www.mecourse.com/landinig/software/cdeconv/cdeconv.html) was used to split the DAB stain and haematoxylin stain colour vectors from RGB images into separate greyscale images, as previously described. ${ }^{1}$ Positive staining was defined using an intensity threshold based on no primary control and positively stained sections (typically intensities $>150$ of 255 on an 8 bit depth). In order to reduce single pixel noise, for platelet counts a size filter was used to remove objects smaller than $0.45 \mu \mathrm{m}^{2}$.

ImageJ particle analysis was then used to count the number of platelets per $\mathrm{mm}^{2}$ of section imaged, as well as the percentage of sample covered by positive staining. During inflammation, neutrophil elastase is released from neutrophils, and neutrophils form bundles during transendothelial migration, so cell segmentation was not possible, and this antigen was quantified using only the percentage of sample fields stained positive.

Spatial association of platelet CD41 immunostaining with neutrophil elastase was investigated using the relate objects module of CellProfiler to make separate counts of CD41+ platelets with any overlap with neutrophil elastase staining and those that did not (CellProfiler cell image analysis software, Broad Institute, http://cellprofiler.org).

\section{Leukocyte and platelet counts from blood microsamples}

Mice were placed in a surgical recovery chamber at $37^{\circ} \mathrm{C}$ for 10 minutes before a $1-2 \mathrm{~mm}$ cut was made with a scalpel blade across the lateral tail vein. Using a micropipette, $2 \mu \mathrm{l}$ of blood was rapidly sampled and diluted 1:100 into Stromatol (Mascia Brunelli) solution for platelet counts, a further $2 \mu \mathrm{l}$ diluted 1:50 in Türk's solution (Merck) for leukocyte counts, and $4 \mu \mathrm{l}$ was taken for a blood smear. Platelet and leukocyte counts were made using hemocytometers and smears were stained with Kwik-Diff stain (Thermo-Fisher) for leukocyte differential counts (200 cells identified per smear).

Flow cytometric analysis of Platelet-leukocyte complex formation.

4 hours post challenge, mice were terminally anaesthetised and whole blood collected via cardiac puncture into citrated syringes. Whole blood was then incubated with an anti-CD41- fluorescein isothiocyanate (FITC) monoclonal antibody for platelets and anti-Ly6G- phycoerythin (PE) monoclonal antibody for neutrophils in saturating concentrations for 20 minutes, followed by lysis of red blood cells. Samples were then analysed on a flow cytometer (FC500; Beckman Coulter) as previously described. ${ }^{2}$ CD41+ events were gated on $\mathrm{Ly}-6 \mathrm{G}^{\text {hi }}$ neutrophils. The percentage 
of leukocytes that are Ly6G ${ }^{\text {hi }}$ neutrophil events attached to CD41+ platelet events. The gating strategy is shown in supplementary figure 7.

Administration of dabigatran etexilate for measurement of ex vivo platelet aggregation, in vivo bleeding time, and processing of lungs for histological analysis.

Groups of mice were treated with $75 \mathrm{mg} / \mathrm{kg}$ Dabigatran Etexilate (Cambridge Bioscience) or vehicle (Saline) control via oral administration at -1 and 24 hours after LPS administration. 48 hours post challenge, mice were anaesthetised with Isoflurane and bleeding times measured by tail tip amputation over 15 minutes. Mice were then culled with whole blood collected via cardiac puncture into citrated syringes, and lungs collected for histology (see below). Platelet rich plasma was isolated from whole blood and pooled according to groups, and stimulated with $1 \mathrm{u} / \mathrm{ml}$ Thrombin and aggregation measured over 10 minutes via light transmission aggregometry in a shaking plate reader (SpectraMax 340PC, Molecular Devices) at $595 \mathrm{~nm}$ at $37^{\circ} \mathrm{C}$.

Lung collection occurred via tracheal cannulation to allow lungs to be inflated in situ with $0.5 \mathrm{ml} 4 \%$ formalin (Sigma, UK), excised and submerged in $4 \%$ formalin (Fisher, UK) for 24 hours. Left lobes underwent dehydration and paraffin wax embedding in a tissue processor over a 20 hour automated cycle (Citadel, UK). Lungs were then sectioned to 7 micrometre thickness using a microtome (Microtom, UK) and loaded onto Superfrost Plus slides (Fisher Scientific, UK).

\section{Detecting platelets in lung tissue}

For immunohistochemistry (IHC), lung sections were cleared in xylene and rehydrated in decreasing concentrations of ethanol before undergoing blocking in $4 \%$ hydrogen peroxide (Honeywell, UK). Sections underwent antigen retrieval in a pressure cooker, using sodium citrate buffer $(\mathrm{pH6.0)}$ for 5 minutes at 100 degrees Celsius before using the CD42b staining protocol described above (Immunohistochemistry using bright field DAB reporter) and quantified as described above (Analysis of images of lung sections).

\section{Bronchoalveolar lavage collection and analysis}

After terminal anaesthesia, 3 washes of $0.5 \mathrm{ml}$ of PBS were flushed into the lung via a tracheal catheter and retrieved in succession. Samples were diluted 1:2 in Türk's solution for leukocyte counts and 1:2 in PBS for cytospin preparation (Cytospin 2, Thermo Fisher Scientific), subsequently dried and stained with Kwik Diff for leukocyte differential counts. 
Platelet factor-4 (PF4, CXCL4) was quantified using the DuoSet mouse PF4 ELISA kit method (R\&D Systems) using BAL supernatant prepared by centrifugation at 1500 rcf for 7 minutes.

1. Ruifrok AC, Johnston DA. Quantification of histochemical staining by color deconvolution. Anal. Quant. Cytol. Histol. 2001;23:291-9.

2. Riffo-Vasquez Y, Somani A, Man F, Amison R, Pitchford S, Page CP. A Non-Anticoagulant Fraction of Heparin Inhibits Leukocyte Diapedesis into the Lung by an Effect on Platelets. Am J Respir Cell Mol Biol. 2016;55:554563. 


\section{Supplementary Figures}

A

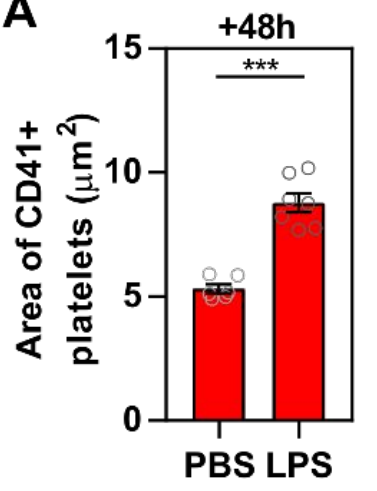

B

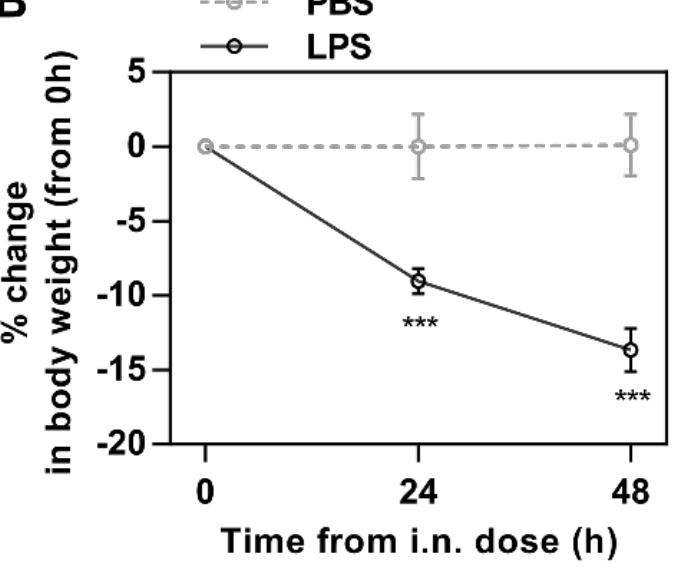

C

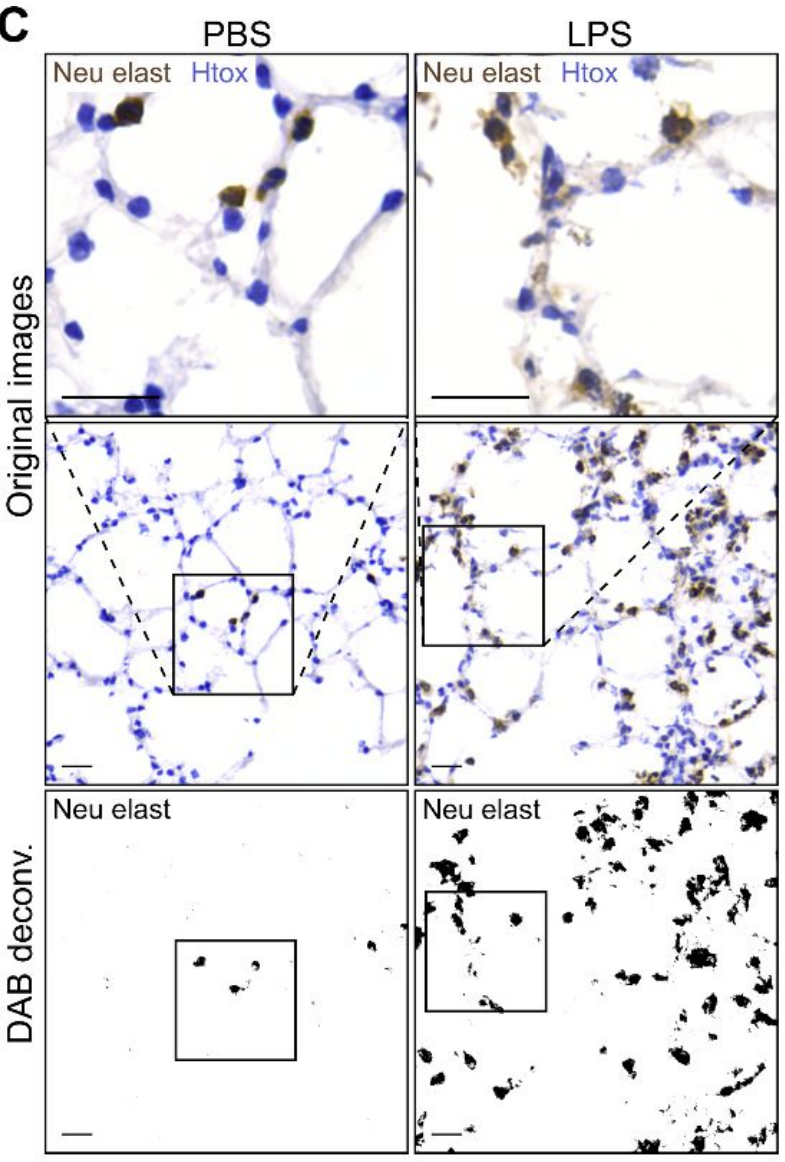

Supplementary figure 1. Effects of LPS inhalation on size of platelets in lung sections, body weight, and lung neutrophil elastase staining using bright field imaging. Mice were administered PBS or LPS $(5 \mathrm{mg} / \mathrm{kg})$ intranasally, and lungs collected at 48 hours. (A) Size analysis of CD41+ events from immunofluorescence quantification. (B) Body weight change from mice used for immunofluorescence experiment, normalized to pre-LPS baseline (Oh). (C) Neutrophil elastase was immunostained in lung sections, and in sample fields CD42b DAB reporter positivity (brown in top row) was deconvoluted (black in bottom row, from same images as top row) for image analysis. Scale bar $=20 \mu \mathrm{m}$. Means \pm standard error, $n=4$, (A) unpaired t-test or (B) 2-way ANCOVA with repeated measures and baseline values fit as covariates and Holm's test for LPS effects within time points, or unpaired t-tests, ${ }^{* * *}=\mathrm{P}<0.001$. 
A

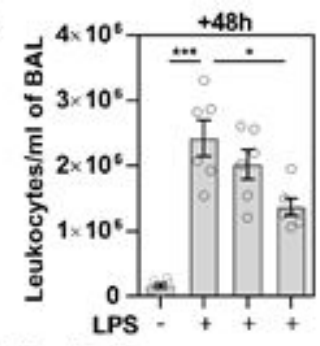

anti-P-selectin - - + - anti-P-selectin anti-PSGL-1 - - - + anti-PSGL-1
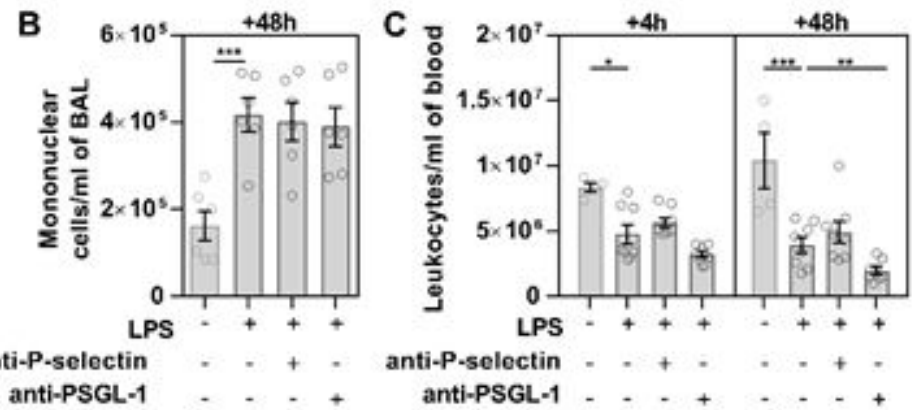

D

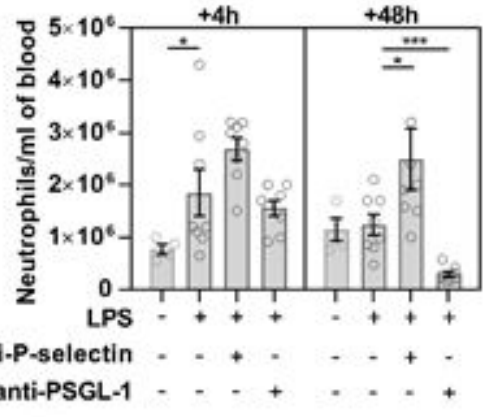

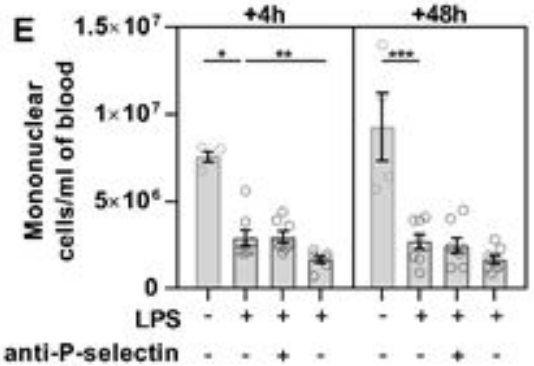

LPS

F

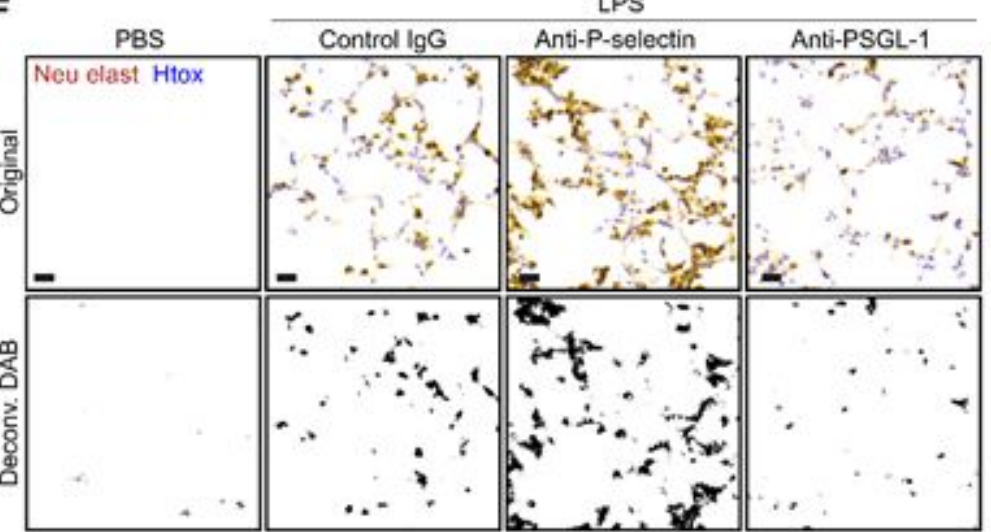

G

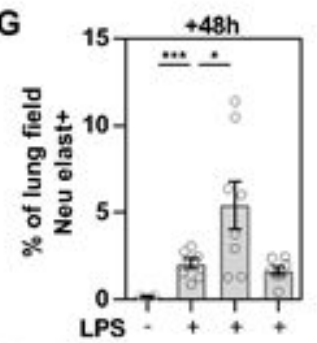

anti-P-selectin . . + anti-PSGL-1

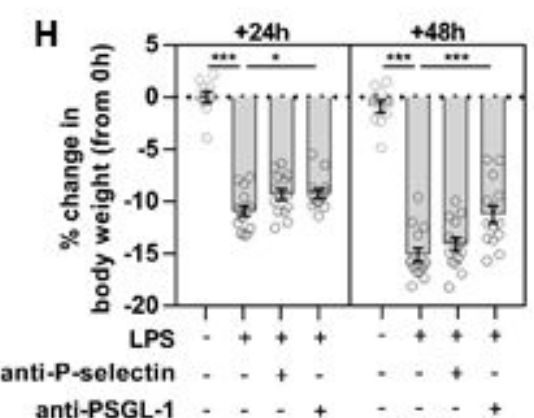

Supplementary figure 2. Effects of P-selectin or PSGL-1 blockade on leukocyte counts in BAL and blood, lung neutrophil elastase staining, and body weight change after LPS inhalation. Mice were administered either control IgG, anti-P-selectin blocking antibody (RB40.34), or anti-PSGL-1 blocking antibody (4RA10) at $4 \mathrm{mg} / \mathrm{kg}$ i.v. in relation to intranasal administration of PBS or LPS $(5 \mathrm{mg} / \mathrm{kg}$ ). (A) BAL leukocyte counts, and (B) mononuclear cell counts. (C) Blood leukocyte, (D) neutrophil and (E) mononuclear cell counts. (F) Lung neutrophil elastase staining and (G) quantification. $(\mathrm{H})$ body weight changes normalised to pre-LPS (Oh) baseline values. Means \pm standard error, neutrophil elastase and blood data, $n=4$ (PBS group) or $n=8$ (LPS groups), BAL data $n=6$, body weight data $n=10$ (PBS groups) or $n=12$ (LPS groups), analyses were (A, B, G) 1-way ANOVA, (C, D, E) 2-way ANOVA with repeated measures, or $(\mathrm{H})$ 2-way ANCOVA with repeated measures and baseline values fit as covariates, all followed by Holm's test for difference vs. LPS + control IgG group, ${ }^{*}=\mathrm{P}<0.05,{ }^{* *}=\mathrm{P}<0.01,{ }^{* * *}=\mathrm{P}<0.001$. 
A

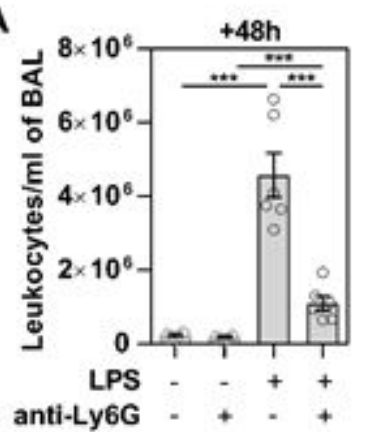

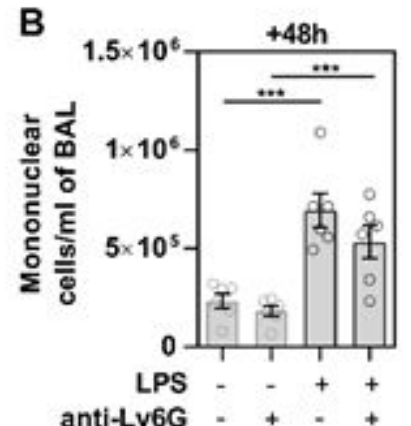
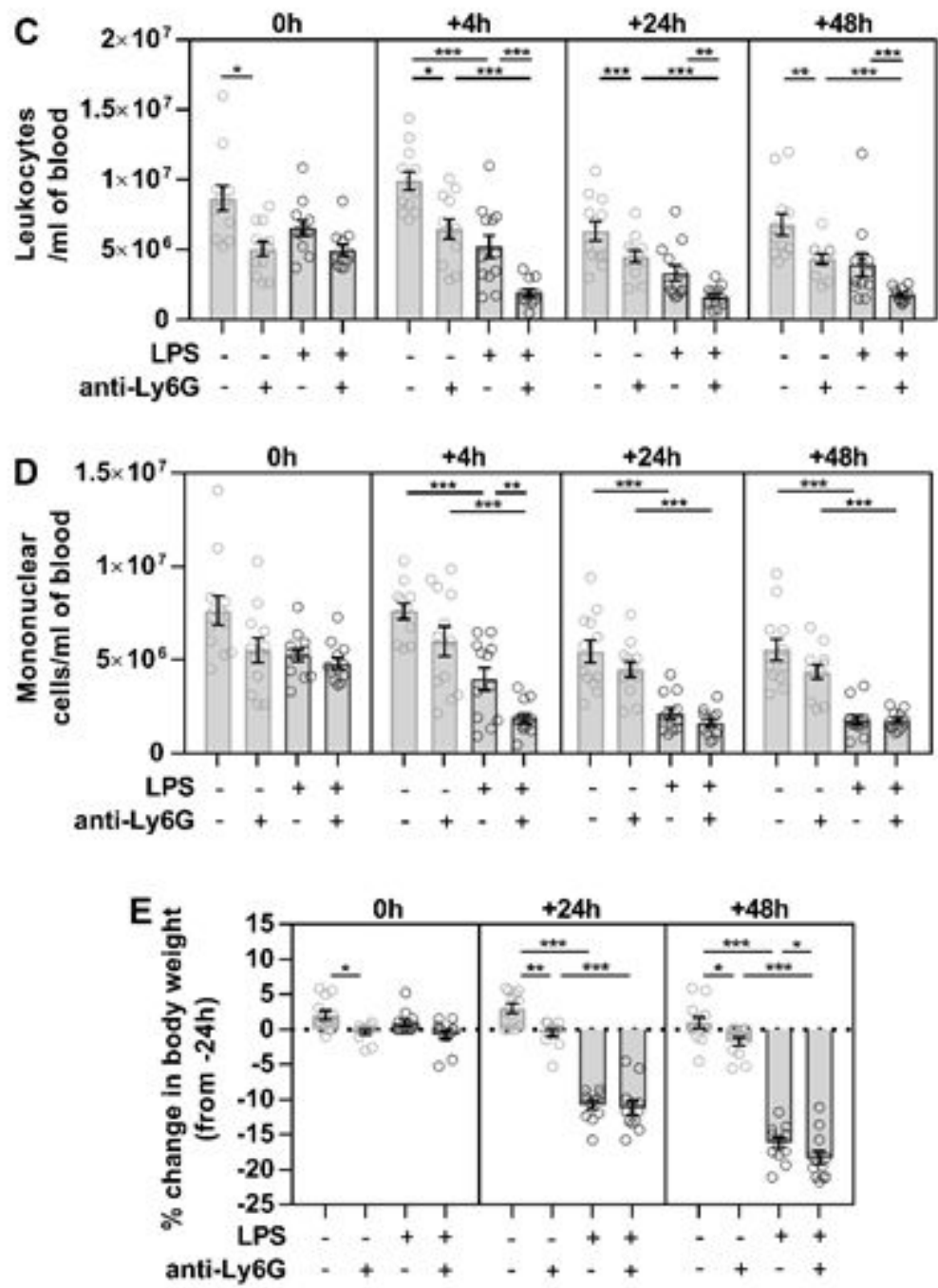

Supplementary figure 3. Effects of LPS challenge and anti-Ly6G antibody treatment on BAL and blood leukocyte counts, and body weight. Mice were administered either control IgG or anti-Ly6G antibody at $-24 \mathrm{~h}$, $-1 \mathrm{~h}$, and $+24 \mathrm{~h}$ in relation to intranasal administration of PBS or LPS $(5 \mathrm{mg} / \mathrm{kg}$ ). (A) BAL total leukocyte counts, and (B) BAL mononuclear cell counts. (C) Blood total leukocyte counts and (D) blood mononuclear cell counts. (E) Body weight changes normalised to pre-antibody administration (-24h) baseline values. Means \pm standard error, (A \& B) $n=6$ or (C, D \& E) $n=12$. Analysis was (A, B \& F) 2-way ANOVA, or (C \& D) 3-way ANOVA with repeated measures, or (E) 3-way ANCOVA with repeated measures and baseline values fit as covariates, all followed by Holm's test for LPS and anti-Ly6G treatment effects, ${ }^{*}=\mathrm{P}<0.05,{ }^{* *}=\mathrm{P}<0.01,{ }^{* * *}=\mathrm{P}<0.001$. 

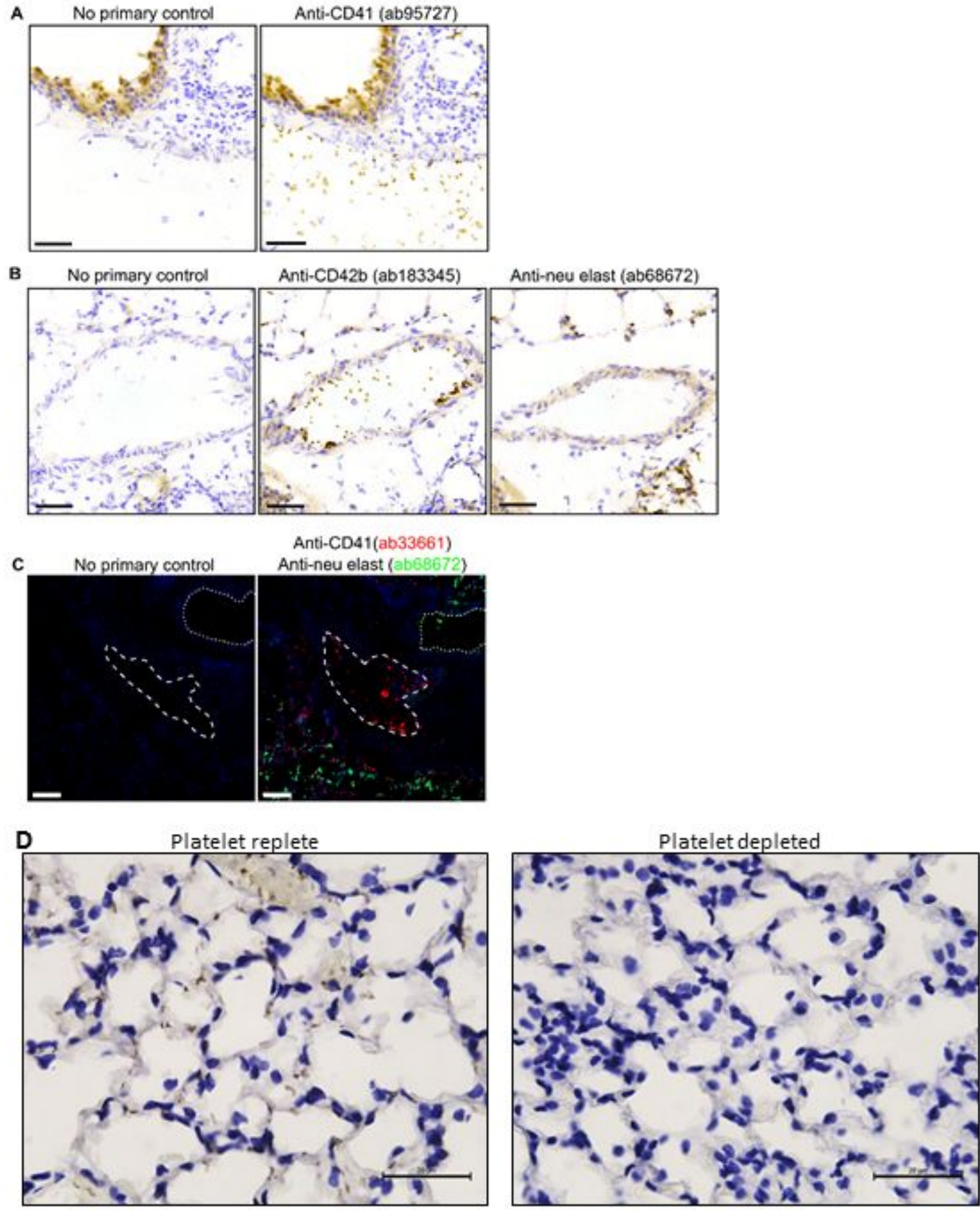

Supplementary figure 4. Additional bright field and fluorescent immunohistochemistry stain validation used to provide evidence for specificity and robustness of platelet and neutrophil staining. Serial sections from LPSchallenged mouse lungs incubated with or without primary antibodies were compared to assess fidelity of immunostaining. DAB reporter positivity in brown, haematoxylin counterstain in blue. (A) CD41 staining using biotinylated rat primary antibody. Single platelets are visible in blood vessel (bottom), false positive staining on respiratory epithelium (top left). (B) CD42b and neutrophil elastase staining using rabbit primary antibodies. CD42b+ platelets are visible in the blood vessel in centre field, neutrophil elastase positivity can be seen in the alveoli-capillary units surrounding. (C) Serial sections from an inflamed lung showing a pulmonary blood vessel (centre, dashed white line for emphasis) with alveolar-capillary network (bottom left) and small airway (top right, dotted white line for emphasis). CD41 is shown as red, neutrophil elastase is in green and nuclei are shown as blue. (D) Mice were administered either control IgG (monoclonal 2A3 anti-trinitrophenol; Bio X Cell) or anti-platelet depleting antibody (anti-CD42b, Emfret Technologies) $25 \mu \mathrm{g}$ per mouse (i.m.) and lungs collected at 24 hours, fixed in formalin and processed to wax-embedded sections. Platelets can be seen stained brown, whereas in platelet depleted mice there is an absence of staining. Both photomicrographs have had their contrast enhanced by $25 \%$ using Microsoft Word formatting feature. Scale bar $=20 \mu \mathrm{m}$. 

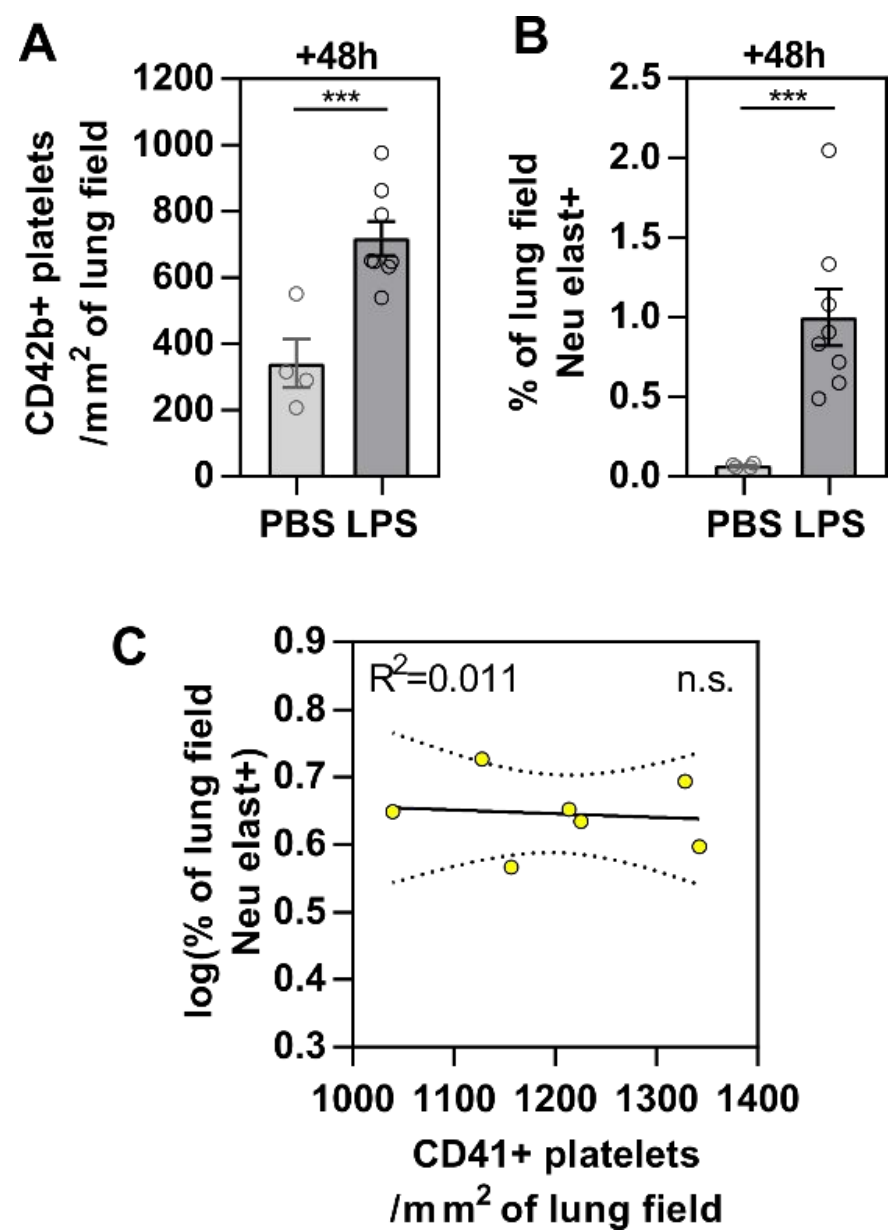

Supplementary figure 5. Additional bright field image analysis data used for correlations, and correlation of lung platelet CD41 and neutrophil elastase levels measured using immunofluorescence. Additional mice were administered PBS $(n=4)$ or LPS $(n=8)(5 \mathrm{mg} / \mathrm{kg})$ intranasally, and lungs collected at 48 hours. (A) Quantification of platelet CD42b immunostaining from bright field images, and (B) the same for neutrophil elastase staining. (C) Correlation of extent of immunofluorescence CD41 platelet staining and neutrophil elastase staining data from the LPS group from Figures 1C \& 1D. Analysis was (A, B) unpaired t-test, or (C) Pearson's correlation, $R^{2}$ and result of Ftest for significantly non-zero slope shown, $* * *=P<0.001$. 
A

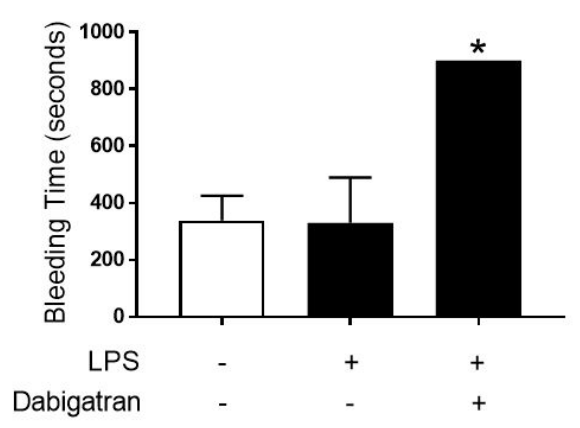

C

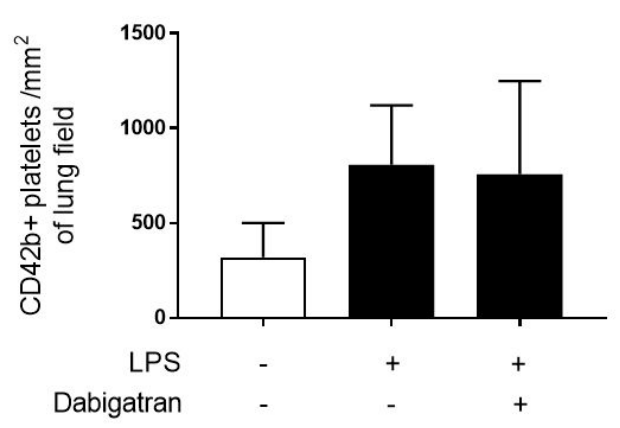

B

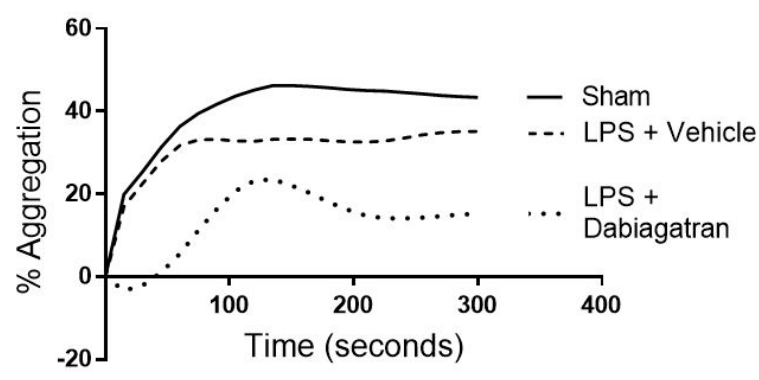

D

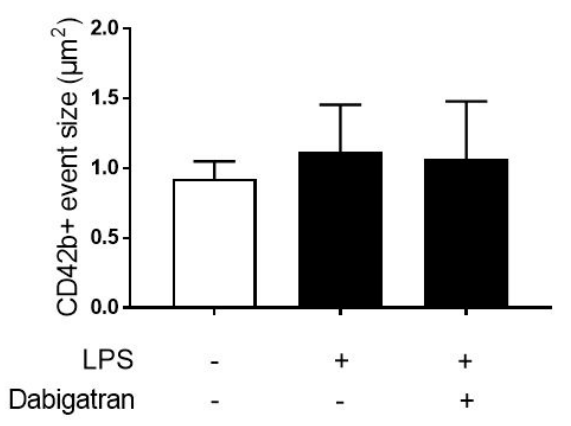

Supplementary figure 6. Effect of a direct thrombin inhibitor (dabigatran etexilate) on haemostatic responses and pulmonary platelet recruitment in response to LPS challenge. Mice were treated with $75 \mathrm{mg} / \mathrm{kg}$ Dabigatran Etexilate or vehicle (Saline) via oral administration at -1 and 24 hours after LPS administration. 48 hours post challenge, (A) bleeding times measured via tail tip amputation, (B) the aggregation of pooled platelet rich plasma of 4 mice towards thrombin assessed; or lungs removed for histological evaluation of the presence of CD42+ platelets (C) per $\mathrm{mm}^{2}$ of lung field, and (D) event size $\left(\mu \mathrm{m}^{2}\right)$. $n=4$ per group. Data represented as means \pm standard error. Data analysed using Kruskal-Wallis with Dunn's test (A) or one-way ANOVA with Dunnett's test (C\&D). ${ }^{* *}=P<0.01$ vs LPS + vehicle group. 
A

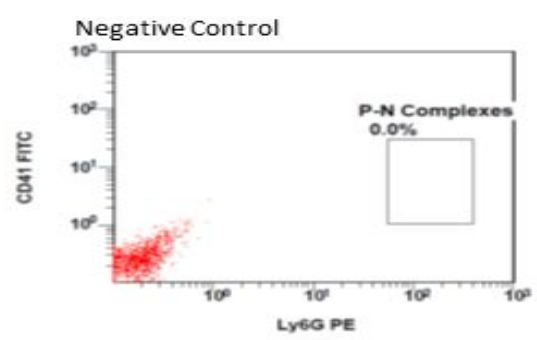

B

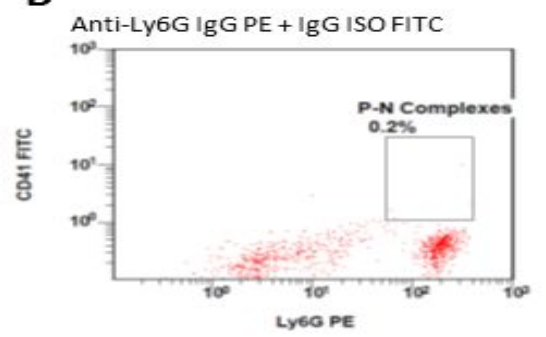

C

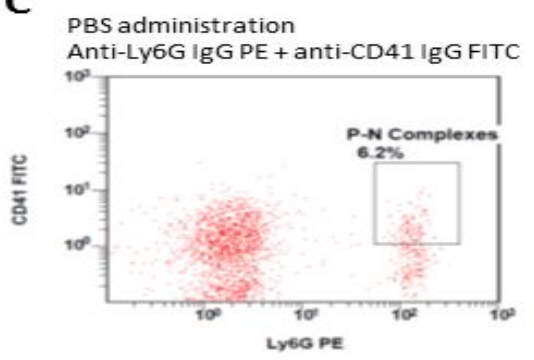

D LPS + control IgG administration Anti-Ly6G IgG PE + anti-CD41 IgG FITC

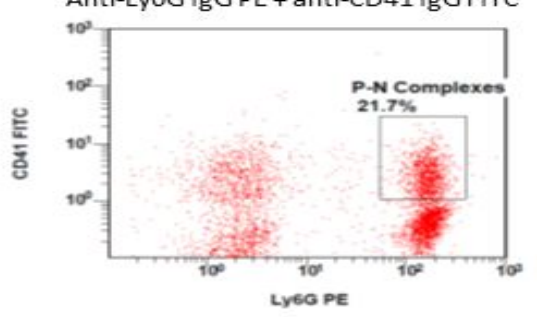

E

LPG + anti-P-Sel' antibody administration Anti-Ly6G IgG PE + anti-CD41 IgG FITC

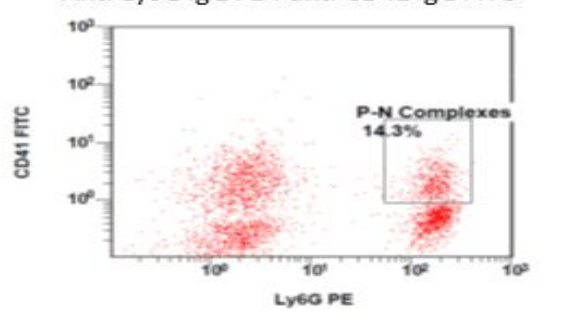

F $\quad$ LPG + anti-P-Sel' antibody administration Anti-Ly6G IgG PE + anti-CD41 IgG FITC

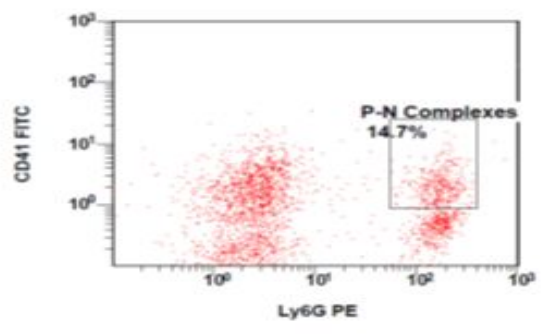

Supplementary figure 7. Gating strategy used to detect the incidence of circulating neutrophil-platelet complexes in blood. Mice were administered either control IgG, anti-P-selectin blocking antibody (RB40.34), or anti-PSGL-1 blocking antibody (4RA10) at $4 \mathrm{mg} / \mathrm{kg}$ i.v. at $-1 \mathrm{~h}$ in relation to intranasal administration of PBS or LPS (5mg/kg). 4 hours later, citrated blood was collected via cardiac puncture and incubated with saturating concentrations of anti-Ly6G-PE and anti-CD41-FITC antibodies for the detection of neutrophil-platelet complexes. (A) representative scatter plot of leukocytes in the absence of antibodies, (B) representative scatter plot of leukocytes incubated with anti-Ly6G-PE, and isotyope control IgG-FITC, (C-F) representative scatter plots of leukocytes incubated with anti-Ly6G-PE and anti-CD41FITC antibodies, revealing the detection of Ly6G hi neutrophils and CD41+ platelets in mice treated with: (C) PBS, (D) LPS + control IgG, (E) LPS + anti-P-selectin blocking antibody, (F) LPS + anti-PSGL-1 blocking antibody. 
Supplementary Movies:

Supplementary movie 1. Demonstration of platelet adhesion tracking method with representative image sequences from a PBS control Pf4-Cre $\times m$ TmG mouse (left) and LPS-challenged Pf4-Cre $\times$ mTmG mouse (right).

Supplementary movie 2. Thromboembolism in pulmonary blood vessels of a Pf4-Cre $\times$ mTmG mouse following injection of thrombin $100 \mathrm{IU} / \mathrm{kg}$ via an intravenous jugular catheter. Scale bar $=\mathbf{5 0} \mu \mathrm{m}$.

Supplementary movie 3. Haemostatic thrombus following traumatic injury to pleural surface of lung of a Pf4-Cre $\times$ mTmG mouse during surgical preparation for lung intravital microscopy. Note thrombi containing spread platelets and adhesive rounded platelets at top left and bottom right of field. Scale bar $=50 \mu \mathrm{m}$.

Supplementary movie 4. Spontaneous capture of a megakaryocyte and subsequent extension of proplatelet projections in the pulmonary circulation of a Pf4-Cre $\times \mathrm{mTmG}$ mouse. Scale bar $=\mathbf{5 0} \mu \mathrm{m}$. 